\title{
Silica optical fiber integrated with two-dimensional materials: towards opto-electro-mechanical technology
}

\author{
Jin-hui Chen ${ }^{1,2}$, Yi-feng Xiong ${ }^{2}$, Fei Xu $\mathbb{C}^{2}$ and Yan-qing $L u^{2}$
}

\begin{abstract}
In recent years, the integration of graphene and related two-dimensional (2D) materials in optical fibers have stimulated significant advances in all-fiber photonics and optoelectronics. The conventional passive silica fiber devices with 2D materials are empowered for enhancing light-matter interactions and are applied for manipulating light beams in respect of their polarization, phase, intensity and frequency, and even realizing the active photo-electric conversion and electro-optic modulation, which paves a new route to the integrated multifunctional all-fiber optoelectronic system. This article reviews the fast-progress field of hybrid 2D-materials-optical-fiber for the optoelectro-mechanical devices. The challenges and opportunities in this field for future development are discussed.
\end{abstract}

\section{Introduction}

Low-loss silica optical fibers, semiconductor lasers and erbium-doped fiber amplifiers lay the foundations of the modern optical communications. In addition to primarily transporting the lightwave, silica optical fibers have found broad applications in the distributed optical sensing ${ }^{1,2}$, endoscope imaging ${ }^{3-5}$, optical trapping ${ }^{6,7}$, fiber lasers ${ }^{8,9}$ and nonlinear optics ${ }^{10}$. With the development of materials science and manufacture technology, the conventional homogeneous doped core and pure cladding structures in a silica fiber have evolved with a new paradigm shift by merging the multi-structures and multi-materials. This emerging trends in optical fiber aim to break the fundamental limit by a single structure and material, and extend their photonic and optoelectronic applications.

In 1978, Hill et al. ${ }^{11}$ demonstrated one-dimensional fiber Bragg gratings (FBGs) employing the photosensitivity in germania-doped fiber. Later, the FBGs have

\footnotetext{
Correspondence: Fei Xu (feixu@nju.edu.cn) or Yan-qing Lu (yqlu@nju.edu.cn) ${ }^{1}$ Institute of Electromagnetics and Acoustics, Xiamen University, Xiamen 361005, China

${ }^{2}$ College of Engineering and Applied Sciences, National Laboratory of Solid State Microstructures and Collaborative Innovation Center of Advanced Microstructures, Nanjing University, Nanjing 210093, China
}

found broad applications in optical communications and sensor systems ${ }^{11,12}$, and have stimulated many other infiber grating structures ${ }^{13,14}$. In the same year, Yeh et al. ${ }^{15}$ proposed the Bragg fiber in which concentric rings of alternating high- and low-refractive index are arranged, to realize lossless propagation in a core of lower refractive index than that of the cladding. From the late 20-century to the dawn of the 21-century, it witnessed booming development of optical fiber technology. Russell et al. ${ }^{16,17}$ successfully combined the concept of two-dimensional (2D) photonic bandgap with the fiber drawing technology, and fabricated the photonic crystal fiber (PCF), which opens a new horizon for in-fiber manipulating optical wavelength, modes, dispersions, polarizations, and nonlinearities ${ }^{18}$. Tong and Knight et al. ${ }^{19,20}$ minimized the width of waveguides and demonstrated the subwavelength silica fiber for low-loss optical waveguiding. The optical microfiber/nanofibers possess many intriguing properties $^{21}$, such as strong field confinement, large evanescent fields and great configurability, and they have been widely used as micro- or nano-scale probes in physical, chemical, biological and materials research ${ }^{21-25}$. Fink et al. ${ }^{26,27}$ focused on integrating multi-materials with disparate electrical, optical, mechanical and thermal properties into

\section{(c) The Author(s) 2021}

(c) (i) Open Access This article is licensed under a Creative Commons Attribution 4.0 International License, which permits use, sharing, adaptation, distribution and reproduction cc) in any medium or format, as long as you give appropriate credit to the original author(s) and the source, provide a link to the Creative Commons license, and indicate if changes were made. The images or other third party material in this article are included in the article's Creative Commons license, unless indicated otherwise in a credit line to the material. If material is not included in the article's Creative Commons license and your intended use is not permitted by statutory regulation or exceeds the permitted use, you will need to obtain permission directly from the copyright holder. To view a copy of this license, visit http://creativecommons.org/licenses/by/4.0/. 


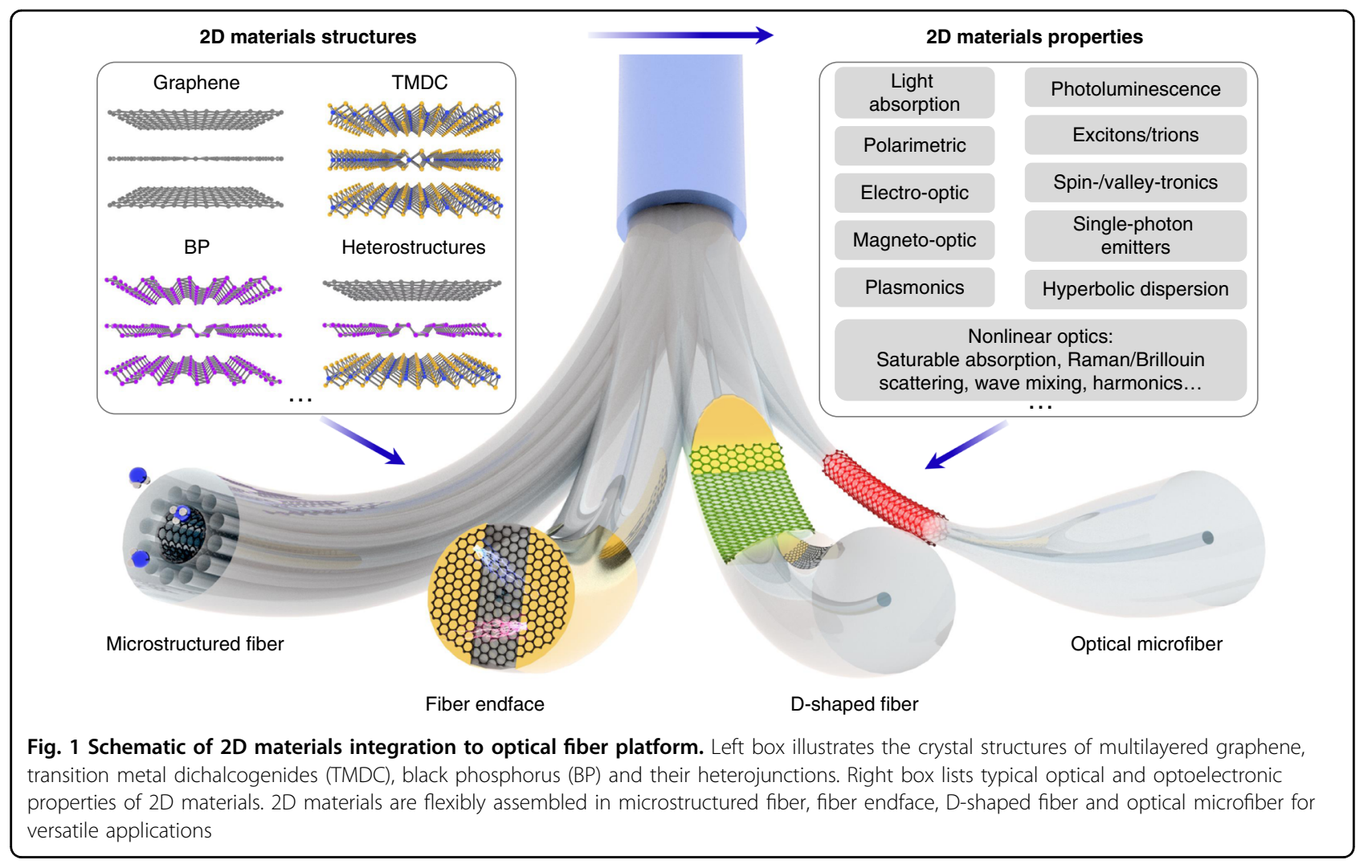

a single fiber, with an ambitious goal of realizing multifunctional fiber devices that see, hear, sense and communicate $^{26,28,29}$. The multimaterial fibers are an important milestone in the development of fiber devices, while it is challenging to seamlessly connect with the universal silica optical fiber networks due to the mode-field mismatch and fiber splicing difficulties.

During this period, the Dirac fermionic graphene in condensed matter physics was emerging rapidly since the seminal work of Geim and Novoselov et al. ${ }^{30,31}$, and it demonstrated many supreme properties such as carrier mobility, thermal conductivity, light absorption, mechanical stiffness/strength and chemical functionalization $^{32-34}$. The rise of graphene and related 2D materials has brought profound impact on nearly every field related to electronics, photonics, chemistry, energy and biology $^{32-37}$. With benefit of hindsight, the 2D materials with salient optoelectronic and mechanical properties are fully incorporated complementarily to the passive silica optical fibers benefiting from their flexibility, configurability and versatility, as shown in Fig. 1. The main advances enabled by $2 \mathrm{D}$ materials are post-processing on the conventional passive silica fiber structures to realize light emission, modulation, switching and detection ${ }^{24,32,38-43}$, which paves the way to all-fiber multifunction-integrated optoelectronics $^{26,29,38}$. The pioneering work of the fiberintegration-2D-materials, such as ultrafast fiber laser ${ }^{44,45}$, graphene polarizer ${ }^{46,47}$, fiber-optic sensor ${ }^{48-54}$ and alloptical modulator ${ }^{55-58}$ have been experimentally realized. Although there is a plethora of comprehensive review papers on 2D materials optoelectronics ${ }^{32,36,38-40,59}$, none of them provide the full pictures and prospects of optical fiber integration. Therefore, we aim to review the fastgrowing research field of hybrid fiber-2D-materials for the opto-electro-mechanical technology. In this article, we first summarize the basic properties of fused silica and three typical 2D materials i.e., graphene, transition metal dichalcogenides (TMDC), and black phosphorus (BP), and particular emphasis is put on the tunability of their linear and nonlinear optical properties. Next, we analyze four kinds of fiber structures integrated with 2D materials (Fig. 1), each of which has their own uniqueness. Then we discuss the all-fiber photonic and optoelectronic applications, i.e. fiber polarizers, light emitting devices, optical modulators, photodetectors, optical sensors and nonlinear optics. Finally, we discuss the challenges and opportunities in the optical-fiber-2D-materials towards the practical applications, and provide our vision for the future perspectives in this field.

\section{Silica and 2D materials properties Silica}

Commercialized standard optical fiber is made of fused silica for their intrinsic ultralow optical loss in the visible 

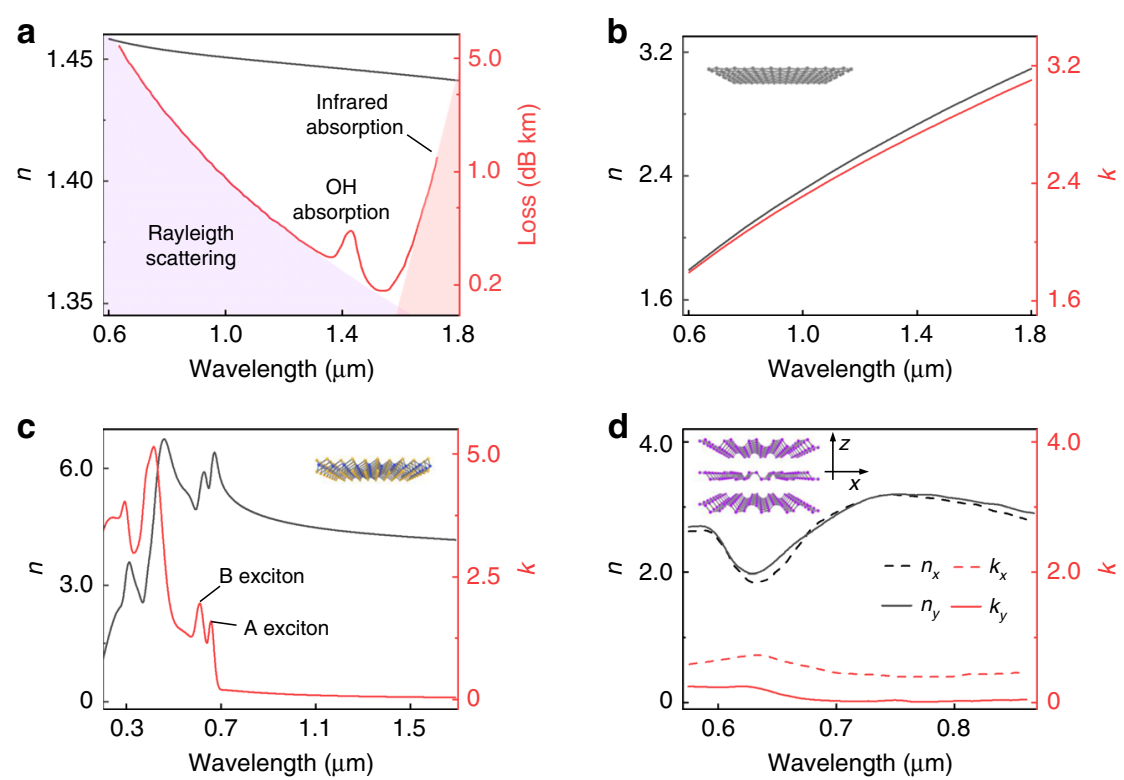

Fig. 2 Optical dispersions of fused silica and 2D materials. a Refractive index $n$ and optical loss (log plot) of silica glass ${ }^{62}$. b Refractive index $n$ and extinction coefficient $k$ of monolayer graphene with the following calculated parameters: Fermi level $0.1 \mathrm{eV}$, scattering time $100 \mathrm{fs}$, temperature $298 \mathrm{~K}$, graphene thickness $0.34 \mathrm{~nm}$. Optical dispersion $(n, k)$ of monolayer $\mathrm{MoS}_{2}(\mathbf{c})^{115}$ and thick BP film with strong anisotropy $(\mathbf{d})^{162}$. The thickness of monolayer $\mathrm{MoS}_{2}$ is set as $0.71 \mathrm{~nm}$. a Reproduced from ref. ${ }^{62}$, with the permission of Springer Nature Switzerland AG 2019. c Reproduced from ref. ${ }^{115}$, with the permission of AIP Publishing. $\mathbf{d}$ Reproduced from ref. ${ }^{162}$, with the permission of OSA Publishing

and near infrared band (Fig. 2a). The first low-loss optical fiber was invented in 1970 at the Corning thanks to the seminal work of Kao and Hockham et al. ${ }^{60}$ who raised the idea that the attenuation in optical fibers caused by impurities could be removed. In current optical fibers, three main loss contributions still exist, i.e., Rayleigh scattering at short wavelength due to the inhomogeneous glass, the infrared lattice vibration and the residual $\mathrm{OH}$ absorption, and the state-of-the-art optical fiber demonstrates propagating loss of $\sim 0.15 \mathrm{~dB} / \mathrm{km}^{61,62}$. Under the regime of linear optics, the isotropic silica has a moderate refractive index of $\sim 1.45$, and the dispersion (Fig. 2a) is well approximated by the Sellmeier formula, which is widely used for characterizing optical materials,

$$
n(\lambda)=\sqrt{1+\sum_{j} \frac{a_{j} \lambda^{2}}{\lambda^{2}-b_{j}}}
$$

where $\lambda$ is the light wavelength, $a_{j}$ and $b_{j}$ are Sellmeier coefficients. As for the nonlinear optics, the primary term of isotropic silica is third-order nonlinearity $\left(\chi^{(3)}\right)$ with the moderate value of $\sim 10^{-20} \mathrm{~m}^{2} / \mathrm{W}$. Due to the ultralong light-matter interaction in silica fiber, efficient nonlinear optics have been revealed, such as Kerr effect, four-wave mixing, stimulated Raman and Brillouin scattering ${ }^{10}$. While under the electric-dipolar approximation the bulk second-order nonlinearity $\left(\chi^{(2)}\right)$ in fused silica is missing, researches show that the structural loss of inversion symmetry at the surface/interface allows second harmonic generation (SHG) with the surface $X^{(2)}$ of the order $\sim 10^{-21} \mathrm{~m}^{2} / \mathrm{V}^{63}$. Further, the electric-quadrupole and magnetic-dipole response also contribute to the SHG response ${ }^{64}$. As for mechanics, the Young's modulus of bulk silica glass is approximately $70 \mathrm{GPa}$. Nevertheless, the measured strength is as low as $0.2 \mathrm{GPa}$ because of the surface imperfections ${ }^{65}$. Intriguingly, Brambilla et al. ${ }^{65}$ recorded the maximum strength of $\sim 26 \mathrm{GPa}$ in silica nanowires with significantly reduced defects.

\section{D materials}

2D materials are a class of crystals whose thickness vary from one-atomic layer to tens of nanometers, and most of them are formed by in-plane covalent bonds and out-ofplane Van der Waals force ${ }^{66}$. The simultaneously high stiffness and elasticity/flexibility in 2D materials enables their adaptation into various photonic structures, enhancing the light-matter interactions. Since the first discovery of graphene, the burgeoning development of materials synthesis has significantly expanded the library of $2 \mathrm{D}$ material from the elements to compounds ${ }^{67-70}$, and more than 600 stable 2D layered materials are predicted ${ }^{71}$. In contrast, only a few mainstay 2D materials are successfully integrated to the optical fiber platform, such as graphene, TMDCs and BP. These 2D materials spans the electronic bandgap of $0-2 \mathrm{eV}$, which corresponds to the optical 
spectral response from the terahertz to visible band, and is sufficient for optical fiber photonics and optoelectronics. There is still huge potential to discover in fiber integration with other novel 2D materials.

\section{Graphene}

As the first discovered 2D materials, the atomic-thin graphene with honeycomb structures has been extensively studied for more than 15 years $^{32-36,38}$, and it still shows strong vitality especially in condensed matter physics so far, for example, the magic-twist graphene layers for superconductor and correlated insulator ${ }^{72,73}$. Conceptually, graphene can be considered as a mother material for fullerene, nanotubes and graphite ${ }^{74}$, while the dimensionality defines their drastic difference. Graphene has an ambipolar electric field effect with carrier mobility reach $10^{6} \mathrm{~cm}^{2} \mathrm{~V}^{-1} \mathrm{~s}^{-1}$ due to its massless Dirac fermions $^{75}$, which is 2-3 orders of magnitude higher than that of the semiconductor silicon, and have found applications in high-frequency transistors ${ }^{76,77}$. The high electron velocity and linear energy-momentum dispersion contribute to graphene's intriguing optical properties, for example broadband light absorption (Fig. 2b). Generally, the linear optical response of graphene can be determined by a surface optical conductivity from Kubo formula, and under the assumption $K_{B} T<<\left|\mu_{c}\right|$, the optical conductivity can be analytically derived as ${ }^{78,79}$

$$
\sigma(\omega)=-\frac{i e^{2} \mu_{c}}{\pi \hbar^{2}\left(\omega-i \tau^{-1}\right)}-\frac{i e^{2}}{4 \pi \hbar} \ln \left(\frac{2\left|\mu_{c}\right|-\left(\omega-i \tau^{-1}\right) \hbar}{2\left|\mu_{c}\right|+\left(\omega-i \tau^{-1}\right) \hbar}\right)
$$

where $k_{B}$ is the Boltzmann's constant, $e$ is the electron charge, $\hbar$ is the reduced Planck's constant, $\tau$ is the relaxation time. The first and second part of Eq. (2) are contributed by the graphene intraband and interband transition, respectively. In the visible and near-infrared spectra with $\hbar \omega>2\left|\mu_{c}\right|$, the interband transition dominates, and the graphene's optical conductivity is $\sim e^{2} / 4 \hbar$, which directly determines the universal light absorption of $\sim 2.3 \%$ per layer ${ }^{80}$. Moreover, the light absorption can be simply tuned by the electric, optical and magnetic field ${ }^{81-84}$, strain gauge $^{85,86}$, and even the molecule adsorption ${ }^{87,88}$. Since the second-order nonlinearity is forbidden in centrosymmetric graphene under the electric-dipole approximation, third-order nonlinearity is the dominant effect with $\chi^{(3)} \sim 10^{-17} \mathrm{~m}^{2} / \mathrm{W}^{44,45,89-}$ ${ }^{91}$. In particular, the broadband, low-threshold-power and ultrafast-response saturable absorption of graphene has attracted great research interest in pulsed fiber laser and all-optical modulation ${ }^{42,43,92,93}$. Manipulating the nonlinear optical absorption is also realized by engineering the Fermi-Dirac distribution ${ }^{94-96}$. Recent experiments systematically reveal the tunable enhancement of third harmonic and four-wave mixing by the Dirac conical bandstructure ${ }^{97,98}$. Note that there is relentless effort in opening graphene's second-order nonlinearity through symmetry breaking, for example, the electric field induced nonlinear effects ${ }^{99}$. High harmonic generation in graphene is observed and enhanced by elliptically polarized light excitation, and this finding sheds light on the possibility of strong field and ultrafast nonlinear dynamics in massless Dirac fermionic materials ${ }^{100,101}$.

The in-plane strong covalent bonds in graphene determine its thermal stability and mechanical strength. Thermally, monolayer graphene is stable in oxygen atmosphere withstanding high temperature of $\sim 300^{\circ} \mathrm{C}$, and the oxidation temperature is up to $500^{\circ} \mathrm{C}$ for multilayers $^{102}$. Mechanically, suspended defect-free graphene shows Young's modulus of $\sim 1.0 \mathrm{TPa}$ and intrinsic strength of $130 \mathrm{GPa}^{103}$, and is highly flexible with a failure strain up to $11 \%^{104}$. The supreme mechanical properties of graphene enable the excellent conformal coating to the optical fiber system.

\section{TMDCs}

TMDCs have a large group of materials with the formula $\mathrm{MX}_{2}$, where $\mathrm{M}$ is a transition metal element from group IV$\mathrm{VI}$ (such as $\mathrm{Mo}, \mathrm{W}, \mathrm{Ti}, \mathrm{Nb}, \mathrm{Zr}$ ) and $\mathrm{X}$ is a chalcogen (such as $\mathrm{S}, \mathrm{Se}, \mathrm{Te}$ ), and there are many comprehensive reviews on TMDCs ${ }^{59,105-107}$. Here we focus on the most studied $\mathrm{MoS}_{2}$ $\left(\right.$ or $\mathrm{WS}_{2}$ ) for its robustness in monolayer limit at room temperature, which benefits for practical optoelectronic devices. Layered $\mathrm{MoS}_{2}$ evolves a transition from indirect bandgap (bulk, $1.2 \mathrm{eV}$ ) to direct bandgap (monolayer, $1.9 \mathrm{eV}$ ) semiconductor due to the lateral quantum confinement effect, and the measured quantum yield of photoluminescence in monolayer crystal is $10^{4}$ higher than that of the bulk crystal ${ }^{108,109}$. The enhanced Coulomb interaction due to the low-dimensional effects in TMDCs forms the tightly bound excitons and trions ${ }^{110-112}$, and they are tunable by electric field and strain gauge ${ }^{113,114}$. Thus, the optical dielectric function of monolayer $\mathrm{MoS}_{2}$ is strongly correlated to the exciton energy ${ }^{115,116}$ in the visible spectra, as shown in Fig. 2c. Moreover, the spin-orbit coupling together with the time-reversal symmetry in monolayer $\mathrm{MoS}_{2}$ leads to valleycontrasting optical dichroism ${ }^{117-119}$, which demonstrates the viability of optical valley control and valleytronics, and finds applications in photonic crystals, plasmonics and waveguides ${ }^{120-124}$. For nonlinear optics, the measured surface second-order nonlinearity in monolayer $\mathrm{MoS}_{2}$ is on the order of $10^{-17}-10^{-19} \mathrm{~m}^{2} / \mathrm{V}^{125-127}$, and the large discrepancy is probably due to the experiment configurations and sample qualities. Researches show that the $X^{(2)}$ nonlinear optics in $\mathrm{MoS}_{2}$ is highly dependent on the layer number ${ }^{125-127}$, stacking order ${ }^{128,129}$, pump wavelength ${ }^{126,130}$, edge state ${ }^{131}$, and even the electrostatic doping ${ }^{132}$. The third-harmonic nonlinear susceptibilities of $\mathrm{MoS}_{2}$ is comparable to that of conventional semiconductors under resonant conditions $\left(\sim 10^{-17} \mathrm{~m}^{2} / \mathrm{W}\right)^{133}$. It is revealed that the few-layer $\mathrm{MoS}_{2}$ 
exhibits significant saturable absorption effects ${ }^{134,135}$, and that monolayer has a strong two photon absorption coefficient as high as $7.6 \times 10^{-8} \mathrm{~m} / \mathrm{W}$, which is three orders of magnitude larger than that of conventional semiconductors ${ }^{136}$.

Mechanically, suspended monolayer $\mathrm{MoS}_{2}$ exhibits Young's modulus of $270 \mathrm{GPa}$ and intrinsic strength of $\sim 23$ $\mathrm{GPa}^{137}$. While the 2D TMDCs can exist in multiple crystal structures with distinct electrical properties, all Mo- and W-based TMDCs except $\mathrm{WTe}_{2}$ are stable in trigonal prismatic phase (hexagonal symmetry) under ambient conditions ${ }^{105,138}$. In addition, the strong electromechanical coupling in TMDCs have enabled structural phase switching by a variety of stimuli, such as chemical doping ${ }^{139}$, mechanical deformation ${ }^{140,141}$ and electrostatic gating ${ }^{142,143}$. The dynamic control of structural phase transition in TMDCs may find applications in phase-change electronic and photonic devices. Recently, the valley-mechanical coupling in monolayer $\mathrm{MoS}_{2}$ is experimentally realized, and it is controlled by pump light, magnetic field gradient and temperature, which paves the way to valley-actuated devices and hybrid valley quantum systems ${ }^{144}$.

\section{Black phosphorus}

Black phosphorus attracts regenerated interest as anisotropic layered materials for electronics and optoelectronics, since it fills the energy gap between semimetallic graphene and semiconducting TMDCs $(1-2 \mathrm{eV})$ with high carrier mobility ${ }^{145-148}$, which is suitable for infrared optoelectronics. Due to the interlayer coupling, the bandgap of BP highly depends on the layer numbers from $0.3 \mathrm{eV}$ (bulk) to $1.7 \mathrm{eV}$ (monolayer) with a power law $E_{\mathrm{opt}}=1.486 / N^{0.686}+0.295$, where $E_{\mathrm{opt}}$ is the optical gap in unit of $\mathrm{eV}$, and $N$ is the layer number ${ }^{149-153}$. Intriguingly, BP always exhibits direct bandgap for various layers, and it is promising for efficient infrared light detection $^{154-156}$ and emission ${ }^{149-151,157}$. The puckered crystal structure endows BP with strong electronic, photonic and mechanical anisotropy ${ }^{145,150,153,154,158,159}$ in contrast to graphene and TMDCs, as shown in Fig. 2d. It is revealed that conductivity along the armchair direction is much higher than along the zigzag direction $^{145,160}$, and the excitons (binding energy $0.3-0.9 \mathrm{eV}$ ) and trions (binding energy $\sim 0.1 \mathrm{eV}$ ) in monolayer BP are also highly anisotropic and robust ${ }^{149,161}$. The linear dichroism in BP (Fig. 2d) can be indicated from the optical selection rule ${ }^{154,162}$, thus their crystalline direction is easily determined through polarization-resolved spectroscopy ${ }^{145,151,154}$. For the nonlinear optics, the third-order nonlinearity of $\mathrm{BP}$ is comparable to graphene and TMDCs, and saturable absorption ${ }^{163,164}$, four-wave mixing ${ }^{165,166}$ and third harmonic generation ${ }^{167,168}$ are observed in BP film.
Regarding mechanics, the theoretical in-plane Young's modulus is $41.3 \mathrm{GPa}(106.4 \mathrm{GPa})$ along the armchair (zigzag) direction in BP, and the sustained strain can be as high as $0.48(0.11)$ along armchair (zigzag) direction owing to the puckered configuration ${ }^{158}$. Tao et al. ${ }^{169}$ measured the Young's modulus of few-layer BP averagely to be $27.2 \mathrm{GPa}$ and $58.6 \mathrm{GPa}$ in armchair and zigzag directions, respectively. The strain effect is extensively researched for anisotropic modulating the electrical and optical functions of $\mathrm{BP}^{170-173}$. Although the bulk $\mathrm{BP}$ is the most stable phosphorus allotrope at room temperature, the few-layer BP is vulnerable to oxygen and water, which hinders their practical applications. In the past years, the stability of BP is comprehensively studied, and many effective passivation techniques are developed, such as the surface encapsulation with $\mathrm{Al}_{2} \mathrm{O}_{3}, \mathrm{SiO}_{2}$ and graphene, and the structural modifications ${ }^{174}$.

\section{D materials fiber integration}

The integrations of $2 \mathrm{D}$ materials to the optical fibers have various architectures based on different materials transfer processes ${ }^{39,88,175}$. According to the light-matter interaction length, the 2D-materials-fiber structures can be categorized into two groups: fiber-endface and guidedwaveguide integration as shown in Fig. 1. The cleaved optical fiber endface is an intriguing platform since it maintains nearly free-space light coupling and manipulation along with remote and self-aligned optical path. Graphene integration to fiber endface is firstly explored for saturable absorption in ultrafast fiber laser for its easy fabrications ${ }^{44,45}$, while suffering from the short lightgraphene interaction length and poor heat dissipation. Researchers usually employed 2D-materials-polymer composites and sandwiched them between two fiber connectors $^{45}$. With shrinking light-based technology, such as plasmonics, photonic crystal and metamaterials/ metasurface, the multi-structures and materials on the fiber endface will promise novel optical fiber optoelectronics $^{176,177}$. When 2D materials integrated on a fiber capillary tip, the unique free-standing diaphragm of atomic thickness enables ultrasensitive all-fiber microelectromechanical system (MEMS) ${ }^{50,51,178}$.

Waveguide integration means that light-matter interaction strength depends on the geometry scale of $2 \mathrm{D}$ materials along the wave propagation, which is free from their atomic-thickness limit. There are mainly three waveguide coupling architectures, i.e. D-shaped fiber (DSF), optical microfiber (MF) and microstructured fiber. In particular, DSF is fabricated either by side-polishing or chemical etching to expose the fiber core and enhance the surface evanescent field ${ }^{179}$, and the flat surface structure is beneficial for excellent contact with grafted 2D materials ${ }^{46,96,180,181}$ as illustrated in Fig. 3a. While less explored, the DSF embedded with nanophotonic structures and 

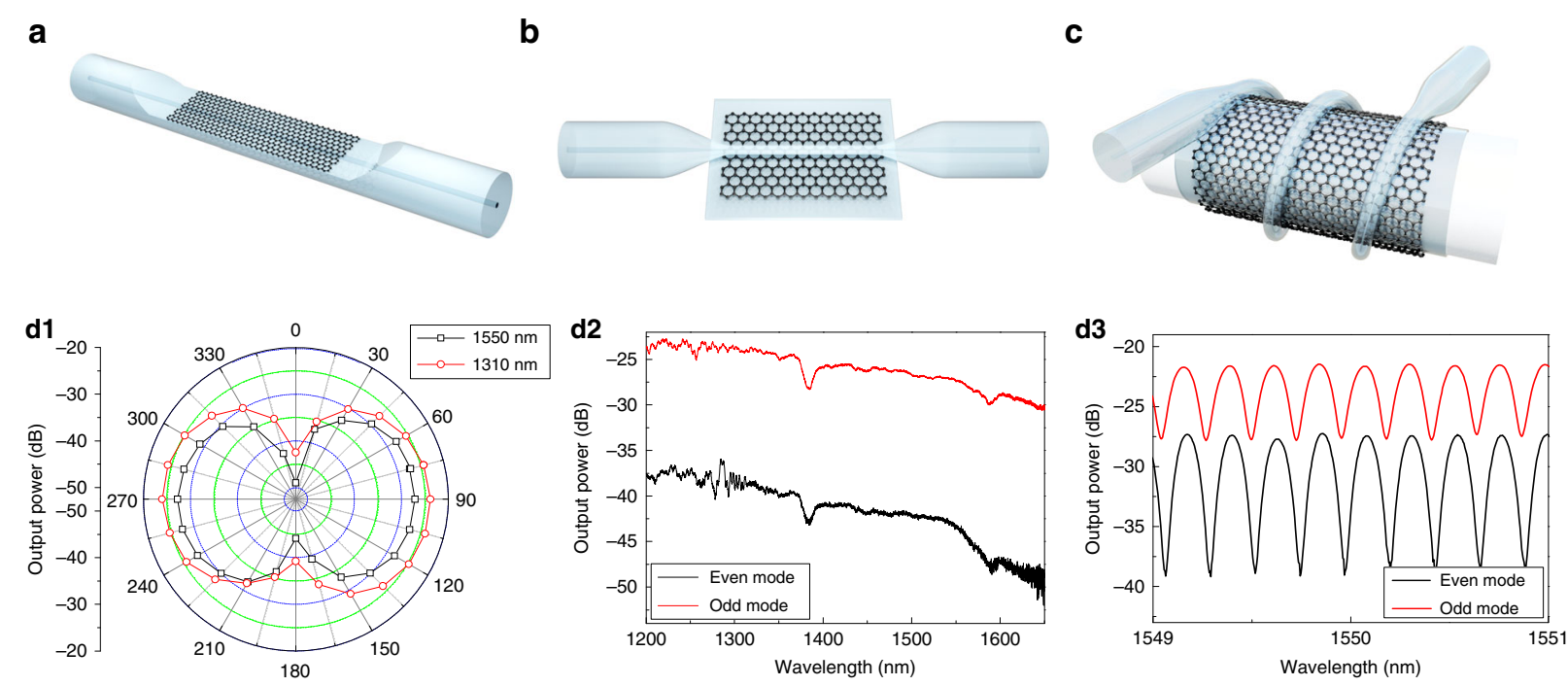

Fig. 3 Polarimetric fiber devices with graphene. a D-shaped fiber laminated with graphene. b Optical microfiber (MF) on graphene-substrate. c Stereo MF-graphene structure with adjacent coil coupled or isolated. Fiber polarizer characterizations in stereo MF-graphene devices by two-coil structure $(\mathbf{d} \mathbf{1}-\mathbf{d} \mathbf{2})^{47} . \mathbf{d} \mathbf{3}$ Transmission spectra of the MF-graphene coil-resonator for two orthogonal modes ${ }^{47} . \mathbf{d} \mathbf{1}-\mathbf{d} \mathbf{3}$ Reproduced from ref. ${ }^{47}$ with the permission of OSA Publishing

functionalized by advanced materials are of great potentials for all-fiber light-manipulation with robustness ${ }^{176,179}$. As for MF integration, the 2D materials, for example graphene, is either wrapped around or line-contacted with an MF (Fig. 1 and Fig. 3b, c). Generally, an MF is continuously tapered or chemically etched from a standard optical fiber $(\sim 125 \mu \mathrm{m})$, the diameter of which ranges from hundreds of nanometers to tens of micrometers $^{21,23,24}$. For subwavelength MF, the large evanescent field enables strong interactions with 2D materials $^{56,182}$, and experimental results show that hybrid MF-WS ${ }_{2}$ of sub-100 $\mu \mathrm{m}$ interaction length is sufficient for $>95 \%$ light absorption ${ }^{182}$. Technically, the surface encapsulated MFs of sub-micrometer diameters are difficult to fabricate and handle, because they are easily broken or contaminated in ambient environment. Xu et al. reported a robust stereo MF-graphene structure with lab-on-a-rod technique ${ }^{47,52,58,183}$. Since the fabrication process only involves laminating a small piece of graphene onto a rod of millimeters diameter, and the MF is helically winded around a surface functionalized rod, arbitrary lightgraphene interaction length can be realized with miniaturized size, as shown in Fig. 3c. Another uniqueness is the formation of optical resonators through intercoupling between adjacent coils for cavity-enhanced interactions ${ }^{47}$. Note that most of the researches focus on the light absorption or spectral shift functions by the fiber-2D-material devices ${ }^{47,55-57,88,184-186}$, the exceptional valleytronics ${ }^{117-119}$, excitonics ${ }^{112,161}$, single photo-emitters $^{187,188}$ and optical-nonlinearity ${ }^{189,190}$ in 2D materials interact with chiral field of $\mathrm{MF}^{191}$ deserves in-depth explorations, which would stimulate all-fiber applications for nanolasers ${ }^{192}$, chiral photonics ${ }^{122}$ and quantum optics ${ }^{193}$.

The microstructured fibers with ingenious microfluidic channels such as photonic crystal fiber (PCF) and hollow core fiber (HCF) are another intriguing platform to accommodate various materials in the hole walls. Indeed, PCF infiltrated with gas, liquids, glasses, semiconductors and metals have significantly extended their functionality in the linear and nonlinear optics ${ }^{194}$. Integrating $2 \mathrm{D}$ materials in PCF is nontrivial since the $2 \mathrm{D}$ materials of atomic thickness not only keep PCF structure and optical functions intact, but also they will perform unique functions that cannot be realized in conventional materials ${ }^{195,196}$. As for the device preparations, solution processed 2D materials are infiltrated into the air hole of PCF or HCF using a pump, and a thin film is deposited after solvent evaporation ${ }^{197,198}$. The solution injection technique is simple, while the quality of infiltrated film is poor, which significantly limits their further applications. Recently, Liu et al. ${ }^{195,199}$ reported a direct chemical vapor deposition (CVD) growth method, and realized massive production of graphene-PCF/HCF with high crystalline quality and environmental adaptability. The breakthrough-work opens new possibilities for scientific research and practical applications in all-fiber optoelectronics.

\section{Photonic and optoelectronic fiber devices}

Motivated by the intriguing physical properties of graphene and related 2D materials, many on-chip monolithic 
photonic and electronic devices are created and developed in the past years ${ }^{36,38,39,41-43,59}$. In contrast, the development of all-fiber photonics and optoelectronics with 2D materials shows much slower pace. This section reviews the mainstream applications of 2D-materials-optical-fiber in the categories of polarizers, light-emitting devices, optical modulators, photo-detectors, optical sensors and nonlinear optics.

\section{Fiber polarizers}

Optical fiber polarizers operate in-line discriminating polarized light transmission with high extinction ratio, which is important in communication, sensor and laser systems. The conventional in-fiber polarizers are based either on asymmetrically polarization-dependent coupling with external materials such as birefringent dielectrics and plasmonic metals, or on single polarization fiber ${ }^{200}$. The graphene-based fiber polarizers have flexible structure designs and tunable functions by electric-gating (Fermi level) ${ }^{46,96,201}$, and there are trade-offs between insertion loss and polarization extinction ratio. Bao et al. ${ }^{46}$, demonstrated ultra-broadband (visible to infrared), high extinction ratio $(27 \mathrm{~dB})$ fiber polarizers on a DSF-graphene structure (Fig. 3a). It is figured that high-order leaky modes with transverse magnetic (TM) polarization suffers larger loss in graphene than with transverse electric polarization (TE), which contributes to the TE-pass polarizer ${ }^{46}$. Later works show that under guided-mode interaction scheme, the passpolarization can be either TE or TM on various waveguide structures ${ }^{47,96,180,202}$, and is fundamentally determined by the in-plane electric field distribution in graphene lay$\mathrm{ers}^{201,203}$. Kou et al. ${ }^{47}$ tailored the stereo graphene-MF structure, and they realized a high extinction ratio fiber polarizer ( 16 dB @ $1550 \mathrm{~nm}$, Fig. 3d1-d2) and high- $Q(2 \times$ $10^{4}$ ) single-polarization fiber resonator (Fig. 3d3) by controlling the near-field coupling between adjacent MF coils. Note that when the adjacent MF coil is decoupled, the stereo-MF-graphene structure is physically equivalent to MF-on-graphene as illustrated in Fig. 3b; since the geometry scale of functionalized $\operatorname{rod}(\sim \mathrm{mm})$ is far larger than MF $(\sim \mu \mathrm{m})$, the spatial curvature and Berry phase can be neglected. Besides graphene and its derivatives, the strong anisotropic 2D materials such as $\mathrm{BP}^{145,154}$ and $\mathrm{ReS}_{2}{ }^{204,205}$ are promising candidates for polarimetric fiber components.

\section{Light-emitting devices}

In principle, 2D materials with direct electronic bandgap are potentially efficient light emitters in the process of excited electrons recombination with holes, and layered $\mathrm{TMDCs}^{108,109,112,206}, \mathrm{BP}^{149,150}$ and their heterostructures $^{207}$ are extensively studied for on-chip light emitting devices. Nevertheless, the high-performance fiber-emitting devices are much less researched. Chen et al. ${ }^{182}$ reported monolayer monocrystalline $\mathrm{WS}_{2}$ transferred to silica MFs, and they observed tunable and strong excitonic photoluminescence (PL) by strain gauge (Fig. 4a1-a2), in contrast to the background spectra from defects and doping in the fiber itself. The enhanced PL are contributed by the near-field light interaction and collection (efficiency $~ 12 \%$ ). Recently, Liao et al. ${ }^{192}$ used a simple photoactivation method to improve the roomtemperature quantum yields of monolayer $\mathrm{MoS}_{2}$ directly grown onto silica microfibers, by more than two orders of magnitude in a wide pump power range, which allows direct lasing with strikingly reduced thresholds down to $5 \mathrm{~W} / \mathrm{cm}^{2}$ (Fig. 4b1-b2). In addition to the classical light generation, the nontrivial single photon emitters (SPEs) in defect or strained 2D material are attracting attentions $^{187,188,208,209}$, and the in-fiber SPEs offer alignmentfree collections and near-resonant excitation schemes. The ideal on-demand SPE emits exactly one photon at a time into a given spatiotemporal mode, and all photons are indistinguishable ${ }^{210}$. Schell et al. $^{211}$ demonstrated coupling of SPEs from 2D hexagonal boron nitride to a tapered MF (Fig. 4c1-c2), and found a collection efficiency of $10 \%$ in the system. The performance of SPEs can be significantly improved by fiber-cavity structures ${ }^{212}$. Exploring 2D materials that generating telecom band $(\sim 1550 \mathrm{~nm})$ SPEs is strongly required in the optical-fiber quantum networks ${ }^{209}$. The ultimate goals of on demand, highly pure, and coherent SPEs integrated with optical fiber remains to be solved.

\section{Optical modulators}

Optical modulators are the essential components in photonics and optoelectronics, which operate at encoding information into the light beams. 2D materials with supreme and tunable photo-response functions by external fields, have driven significant advances of optical modulators, and there are several comprehensive reviews in this topics ${ }^{42,43,213}$. Here we focus on the fibercompatible modulators. The all-optical modulator (AOM) that uses one light beam to control the transmission of another one, can realize ultrafast modulation speed avoiding the electrical bottleneck. In principle, the AOM based on Pauli-blocking effect ${ }^{55,56,58,180,214,215}$, Kerr effect $^{216,217}$ and opto-thermal effect ${ }^{57,218-221}$ are widely studied in various 2D materials such as graphene, BP and TMDC $^{42,43,213,222-224}$. Liu et al..$^{55}$ first reported broadband all-optical modulation using a graphene-coveredmicrofiber (GMF) structure. Later, Li et al. ${ }^{56}$ pushed the response-time of GMF to the carrier-relaxation limit of graphene $\sim 2.2$ ps though with a small modulation depth (MD) of $\sim 1.4 \mathrm{~dB}$, as shown in Fig. 5a1-a2. It is challenging to fabricate and manipulate such sub-wavelength GMF $(\sim 1 \mu \mathrm{m})$ for practical applications. Chen et al.$^{58}$ realized a robust stereo GMF structure (Fig. $5 \mathrm{~b}$ ) for polarizationdependent light modulation with a maximized MD of 

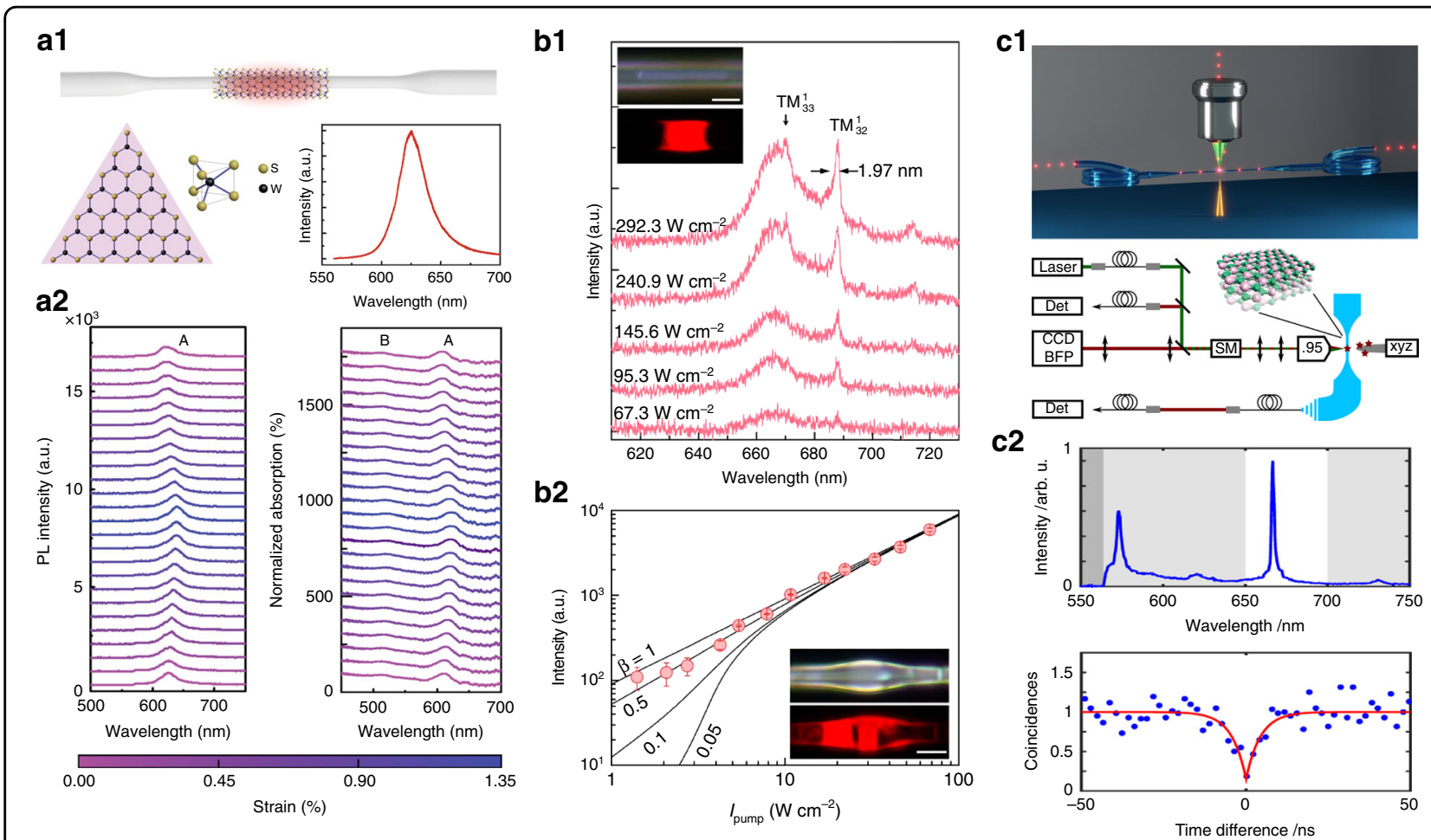

Fig. 4 Light emitters integrated in optical microfiber. a1 Schematic hybrid MF-WS 2 structure for enhanced light emission ${ }^{182}$. Inset: Crystal structure of a triangular monocrystalline $\mathrm{WS}_{2}$ (left panel), and photoluminescence (PL) spectra of a monolayer $\mathrm{WS}_{2}$ (right panel) ${ }^{182}$. a2 $\mathrm{In}$-line strain manipulation of the PL (left panel) and absorption spectra (right panel) of hybrid MF-WS ${ }_{2}^{182}$. Room-temperature continuous wave lasing from asphotoactivated monolayer $\mathrm{MoS}_{2}$ in MF resonator (b1) and microbottle resonator (b2) ${ }^{192}$. c1 Coupling quantum emitters of hBN with MF waveguide $^{211}$. c2 Spectra of the light collected through the fiber (top), and anti-bunching measurements of the light collected through the fiber (bottom) ${ }^{211}$. a1-a2 From ref. ${ }^{182}$. Reproduced by permission from Springer Nature: Light: Science \& Applications. b1-b2 Reprinted by permission from AAAS $^{192}$. $\mathbf{c 1 - c 2}$ From ref. ${ }^{211}$. Reprinted by permission from ACS Publications

$\sim 7.5 \mathrm{~dB}$ and a modulation efficiency of $\sim 0.2 \mathrm{~dB} / \mathrm{mW}$. Gan et al. ${ }^{57}$ demonstrated an all-fiber phase shifter assisted by graphene's photothermal effect, and they obtained a phase shift exceeding $21 \pi$ with a maximized slope of $0.192 \pi /$ $\mathrm{mW}$, as shown in Fig. 5c1-c3. Towards the practical applications, the performance indexes of fiber AOM such as the control power consumption, switching time, MD and insertion loss need to be globally optimized and balanced $^{225}$. For example, the larger MD generally requires enhanced light-matter interactions either through field confinement or interaction length, which often brings higher insertion loss from materials absorption and scattering; the geometry scale of device, such as waveguide diameter and length may also influence the ultimate switching time ${ }^{42}$.

The electro-optic modulators (EOM) implementing electric field to control the light properties are particularly desirable in current communication networks. Although there have been substantial achievements for on-chip EOM with graphene ${ }^{42,43,213,226}$, based on the tunable electroabsorption or electro-refractive effects. It is nontrivial to develop high performance all-fiber $\mathrm{EOM}^{227,228}$ for their seamless connection to the mainstay optical fiber systems. $\mathrm{Xu}$ et al. ${ }^{227}$ proposed a high-speed traveling-wave EOM on a graphene/MF structure with a $3 \mathrm{~dB}$ bandwidth of $82 \mathrm{GHz}$, as shown in Fig. 5e. Experimentally, Lee et al. ${ }^{96}$ demonstrated ion liquid gating $(\sim 3 \mathrm{~V})$ in multilayered-grapheneDSF with MD of $\sim 10 \mathrm{~dB}$ for TE polarization. Liu et al. ${ }^{195}$ reported a graphene-PCF EOM with large MD of $\sim 20 \mathrm{~dB} /$ $\mathrm{cm}$ under $\sim 2 \mathrm{~V}$ gate voltage. These work use ion liquid as efficient gating medium while suffering low modulation speed and long-term stability. For high-speed modulations, the adaptation of solid gating-dielectric (Fig. 5d-e), sophisticated circuit design and 2D-materials engineering in fiber EOM are worth of further research ${ }^{226,229,230}$.

Besides the aforementioned modulating configurations, the acoustic-optic, mageto-optic, elastic-optic, electromechanical and valley-optomechanical effects in 2D materials are potential candidates for optical modulators $^{42,43,144,231}$. For example, the unique elastic-optic response in graphene has enabled mechanical intensitymodulation in the GMF structure with an MD of $\sim 0.04 \mathrm{~dB} /$ $\mathrm{mm}$ under $1 \%$ strain (MF diameter of $5 \mu \mathrm{m}$ ), and the modulation rate can reach hundreds of kilohertz ${ }^{184}$. 

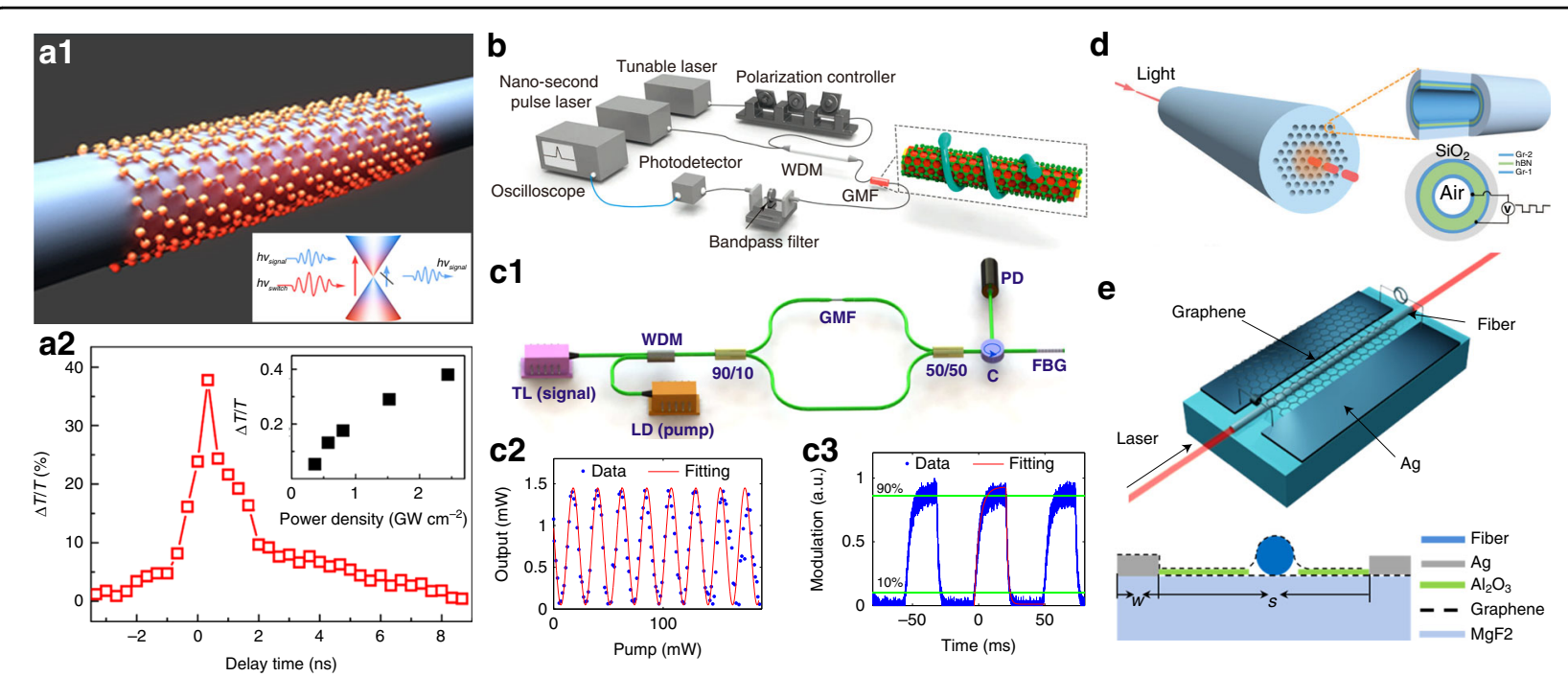

Fig. 5 Optical fiber modulators integrated with 2D materials. a1 Schematic illustration of a graphene-coated microfiber (GMF) structure for alloptical modulator. Inset: Schematic describes Pauli blocking effect ${ }^{56}$. a2 Pump-probe delay time measurement results. Inset: Relation of the modulation depth and pump intensity ${ }^{56}$. b Measurement setup of the pump-probe system with a stereo graphene-MF device ${ }^{58}$. Experimental setup for measuring the phase shift in GMF (c1), all-optical switching by a GMF (c2), and temporal response of the all-optical switching $(\mathbf{c} 3)^{57}$. $\mathbf{d}$ Schematic sketch of a sandwiched graphene/hBN/graphene photonic-crystal-fiber electro-optic modulator (EOM) ${ }^{229}$. e Schematic diagram of the MF-based EOM (upper panel) and its cross-section structure (lower panel) $)^{22}$. a1-a2 Reproduced from ref. ${ }^{56}$ with permission of ACS publications. b Reproduced from ref. ${ }^{58}$ with permission of Springer Nature: Light: Science \& Applications. c1-c3 From ref. ${ }^{57}$. Reprinted by permission of OSA Publishing. d Reproduced from ref. ${ }^{229}$ with permission of The Royal Society of Chemistry. e Reprinted from ref. ${ }^{227}$ by the permission of IEEE Publishing

\section{Photo-detectors}

Photodetectors convert light signals into electrical signals that can be processed by standard electronic circuits. Conventionally, the in-fiber optical signals are out-coupled and detected by external planar photodetectors, which are fabricated on silicon or other bulk semiconductors. The development of 2D materials brings new possibilities to realize all-fiber photodetectors (FPD), since they are of broad photo-response spectra and highly mechanical flexibility without any need of epitaxial substrate ${ }^{232,233}$. Sun et al. $^{234}$ demonstrated broadband (1500 nm-1600 nm) photodetection in a microfiber-graphene photoconductive device, while the photocurrent responsivity is as small as $\sim 2.81 \mathrm{~mA} / \mathrm{W}$ as shown in Fig. 6c1-c2. Chen et al. ${ }^{235}$ fabricated visible-light response FPD by directly bonding fewlayer $\mathrm{MoS}_{2}$ to a fiber endface along with paired gold electrodes. Furthermore, they employed Van der Waals heterostructures to improve device performances ${ }^{236-238}$. For example, using multilayer graphene- $\mathrm{MoS}_{2}-\mathrm{WS}_{2}$ with layer-by-layer transfer method, an ultrahigh responsivity of $6.6 \times 10^{7} \mathrm{~A} / \mathrm{W}$ (Fig. 6a1-a2) and a time response of $\sim 7 \mathrm{~ms}$ at $400 \mathrm{~nm}$ light wavelength were achieved. The sub-band transitions and photogating effect in the heterostructures enable broadband spectra detection ranging from 400-2000 nm with high responsivity (Fig. 6a3) ${ }^{236}$. Recently, Zhuo et al. $^{239}$ assembled a hybrid carbon nanotubes/graphene on a DSF, and they realized a maximized photoresponsivity of $\sim 1.48 \times 10^{5} \mathrm{~A} / \mathrm{W}$ (Fig. 6b1-b2) ${ }^{239}$. Jin et al. ${ }^{240}$ developed a clean device transfer technique and realize near-field coupled $2 \mathrm{D}$ InSe photodetectors on surface of a multimode fiber with fast response time $(\sim 67 \mu \mathrm{s})$. A proof-of-concept binary image transmittance and detection by the InSe FPD was demonstrated, as shown in Fig. 6d. Note that given the figures-of-merit in photodetectors $^{232,233,241}$, i.e. responsivity, electrical/optical bandwidth and noise equivalent power, there are still many technical issues to be solved in FPD devices compared with the on-chip photodetecting architectures ${ }^{232,241}$, since the sophisticated micro-/nano-fabrication technology and diversified 2D materials transfer/processing are stringently lacking in current optical fiber platform. Extending 2D library, combining nanophotonic structures and advancing electrical designs in fiber-endface and DSF platforms are exciting areas to be explored ${ }^{176,230,232,233,239,241-243}$.

\section{Optical sensors}

The optical sensors transform environmental stimuli into the modulated light signal, which is widely implemented for the ever-growing demand of Internet of Things ${ }^{88,244}$. Generally, the 2D-material-integrated optical fiber sensors hold high sensing performance considering the fact that their optical responses are easily modulated by the external stimuli $^{87,245-247}$. In the last few years, researchers have developed various hybrid-fiber schemes for physi$\mathrm{cal}^{52,184,248,249}$ and chemical sensing $24,88,244,250-252$. In particular, graphene and related $2 \mathrm{D}$ materials are appealing 


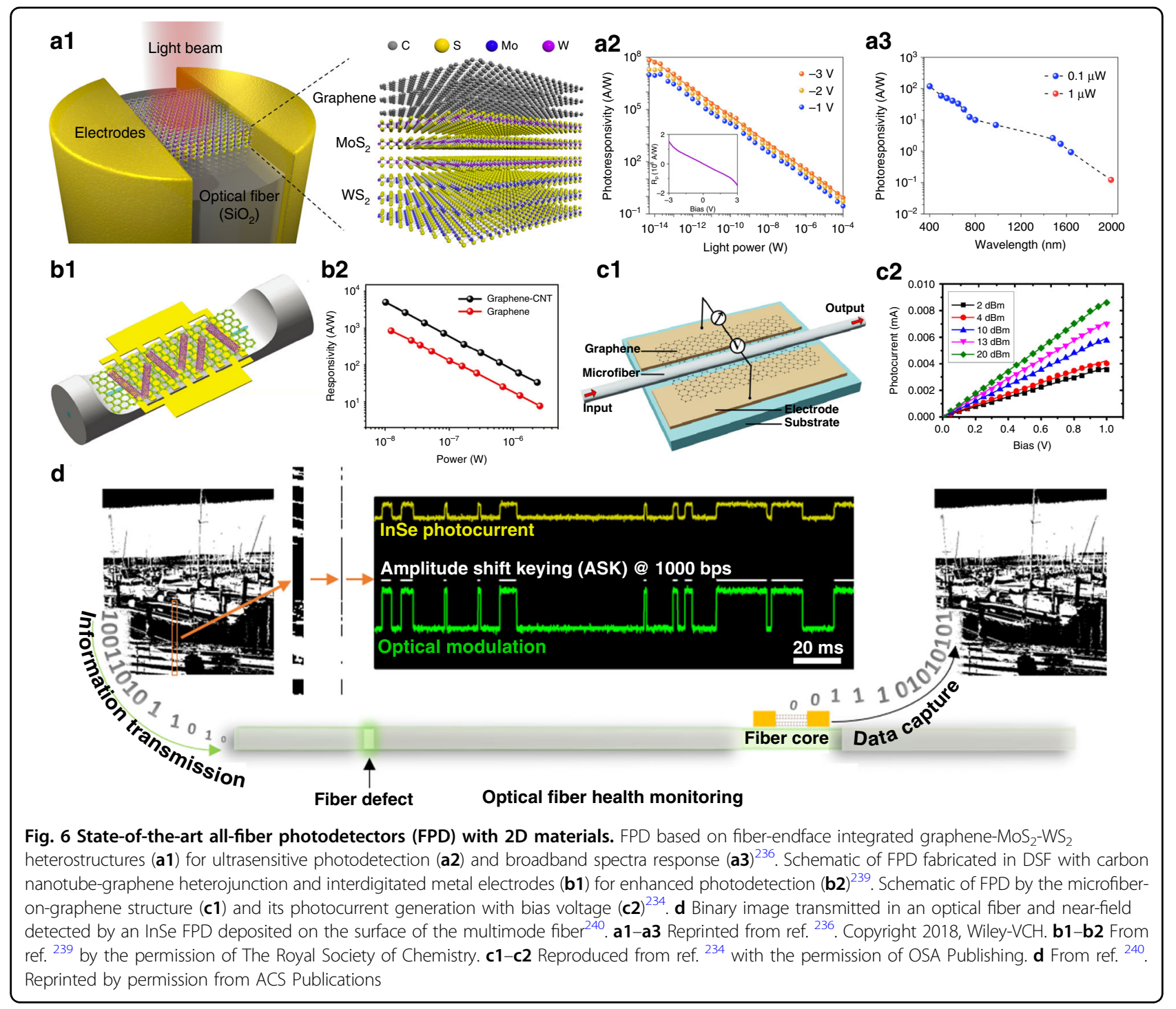

platform for chemical molecules sensing since they have ultimate surface-to-volume ratio, large adsorption capacity and ultrafast carrier mobility ${ }^{88,244,253}$. In principle, the adsorption of molecules changes the permittivity of the 2D materials, which in-turn modulates the parameters of coupling light source, i.e. amplitude, phase, polarization and wavelength ${ }^{24,88}$. The guided evanescent wave in either MF or DSF waveguides ${ }^{88,251,252,254}$ with the fiber grating $^{48,255,256}$, interferometer ${ }^{257,258}$ and microresonator ${ }^{52,259}$ structures are widely studied to increase the sensor sensitivity and reduce the detection limit. For example, Wu et al. ${ }^{48}$ reported a graphene-coated MF Bragg-grating for sensitive gas sensing as shown in Fig. 7a1-a2, and the obtained sensitivities are $4 \mathrm{pm} / \mathrm{ppm}$ and $2 \mathrm{pm} / \mathrm{ppm}$ for ammonia and xylene gas, respectively. Hao et al. ${ }^{260}$ demonstrated graphene-based ammonia sensor using an in-fiber Mach-Zehnder interferometer with a sensitivity of $\sim 3 \mathrm{pm} / \mathrm{ppm}$. Note that most of the work are based on graphene, which often suffer from the cross-talk and limited selectivity problems, it is promising to explore other 2D materials, heterostructures or surface functionalizations to achieve high-selectivity label-free sensors $^{244,261-263}$. Besides the aforementioned passive sensing, Cao et al. recently demonstrated grapheneenabled fluorescent resonance energy transfer in fibermicrofluidic resonator for ultrasensitive and selective biochemical detection, as shown in Fig. 7b1-b2 ${ }^{53,264}$. They achieved individual-molecule sensitivity for dopamine, nicotine and single-strand DNA detection through dual amplifications from optical pump and electrical locked-in detection ${ }^{53}$. An et al. ${ }^{54}$ achieved individual gas molecule detection employing electrically tunable fourwave-mixing effects in graphene bipolar-junctiontransistor heterogeneous DSF, as shown in Fig. 7c1-c2.

The atomic-layer thickness of 2D materials with supreme mechanical properties enables high-performance MEMS 


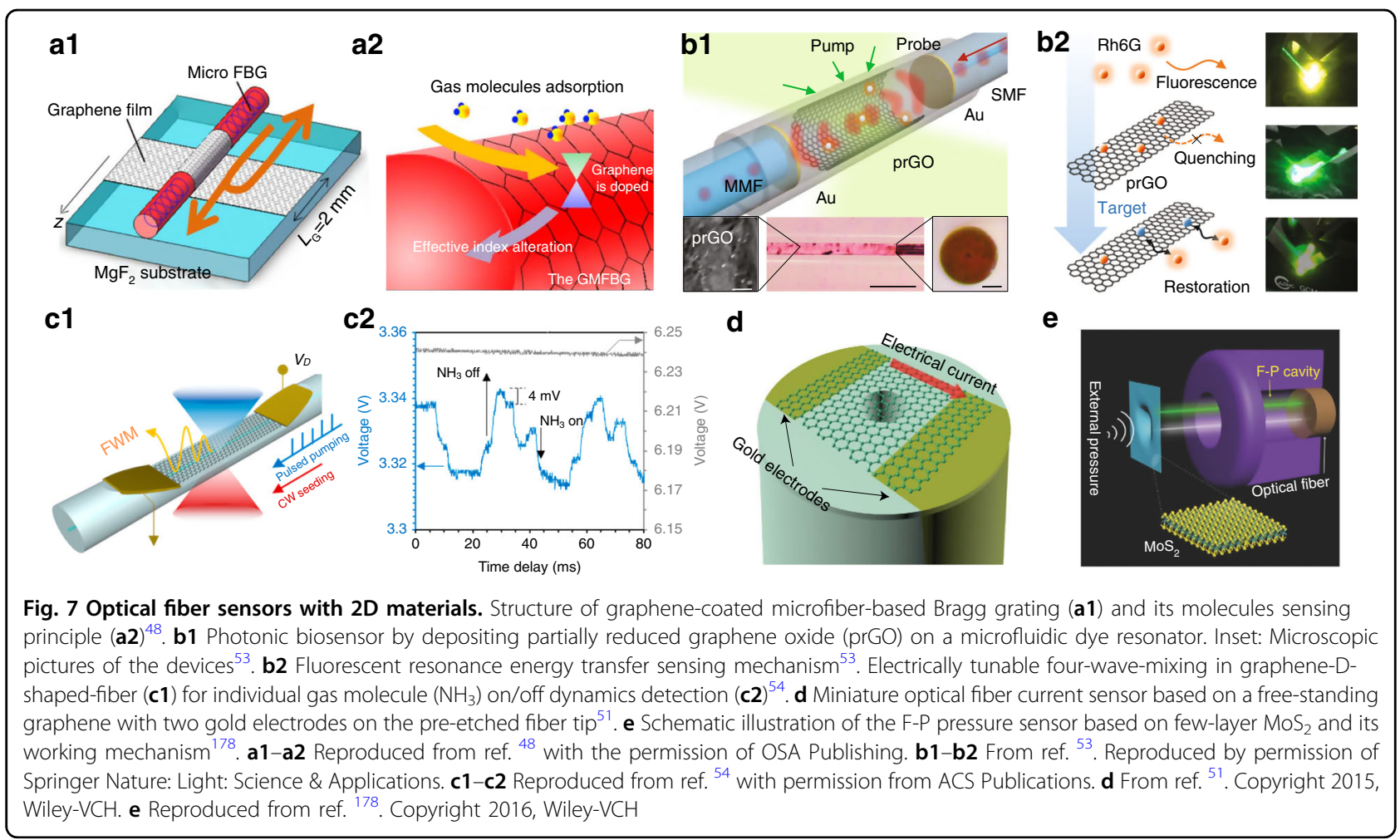

for fiber sensing applications ${ }^{50,138}$. Figure $7 \mathrm{e}$ shows the typical structure of a fiber-integrated $\mathrm{MoS}_{2}$-MEMS sensors, in which the free-standing $\mathrm{MoS}_{2}$ diaphragm and fiber endface form a Fabry-Perot interferometer ${ }^{178}$. The external stimuli deform the $\mathrm{MoS}_{2}$ membrane and change the cavity length, which shifts the optical interference spectra. The relation between the deflection of the diaphragm and external pressure could be modeled as ${ }^{178}$ :

$$
P=\frac{A \sigma_{0} t \Delta L}{r^{2}}+\frac{B E t \Delta L^{3}}{(1-v) r^{4}}
$$

where $A$ and $B$ are dimensionless coefficients, $P$ is the applied pressure, $\sigma_{0}$ is the pre-stress, $r$ and $t$ are the radius and thickness of a circular diaphragm, $\Delta L$ is the center deflection of diaphragm exposed to the pressure, $E$ and $v$ are materials Young's modulus and Poisson's ratio respectively. Ma et al..$^{50}$ first reported a miniature fibertip pressure sensor using a few-layer graphene as a diaphragm, and they observed a spectra sensitivity over $39.4 \mathrm{~nm} / \mathrm{kPa}$. Later, higher pressure sensitivity is achieved in $\mathrm{MoS}_{2}$ diaphragm sensors, as anticipated by their reduced Young's modulus and improved film quality (Fig. 7e $)^{178}$. Zheng et al. $^{51}$ demonstrated ultrasensitive $\left(2.2 \times 10^{5} \mathrm{~nm} / \mathrm{A}^{2}\right)$ and fast-response $(\sim 0.25 \mathrm{~s})$ electrical current sensor by depositing both gold electrodes and graphene membrane on an etched fiber tip, as shown in Fig. $7 \mathrm{~d}$. The highly efficient and localized ohmic-heating, and high thermal conductivity in graphene film synergistically contribute to the high-performance sensors. Besides the quasi-static deformations, the intrinsic nanomechanical resonators by clamped 2D materials also allow the development of vibrational fiber-optic sensors for robust force, mass and pressure measurements ${ }^{265,266}$. Note that both the resonating frequency and quality factor of $2 \mathrm{D}$ materials in the fiber platform are far less than the on-chip devices ${ }^{267,268}$, and further research are needed to optimize the $2 \mathrm{D}$ materials geometry and manipulate the pre-stress in fiber devices.

\section{Nonlinear optics}

Nonlinear optics is the study of the phenomena that optical response of materials are modified by the light field, and it has found broad applications in novel light source generating, signal processing and optical imaging. The state of art 2D materials have enabled many scientific advances in nonlinear effects, such as saturable absorption, Kerr effect, harmonic generation and parametric oscillation $^{93,189}$. In particular, the saturable absorbers (SA) that realize high (low) transmittance of high (low) power density beam, are comprehensively studied in 2D materials for pulse laser generation, of which the laser wavelength spans from visible to the mid-infrared and the pulse width ranges from microsecond to subpicosecond $^{92,93,269-271}$. For example, Bao and Sun et al. $^{44,45}$ pioneered the study of the graphene mode- 


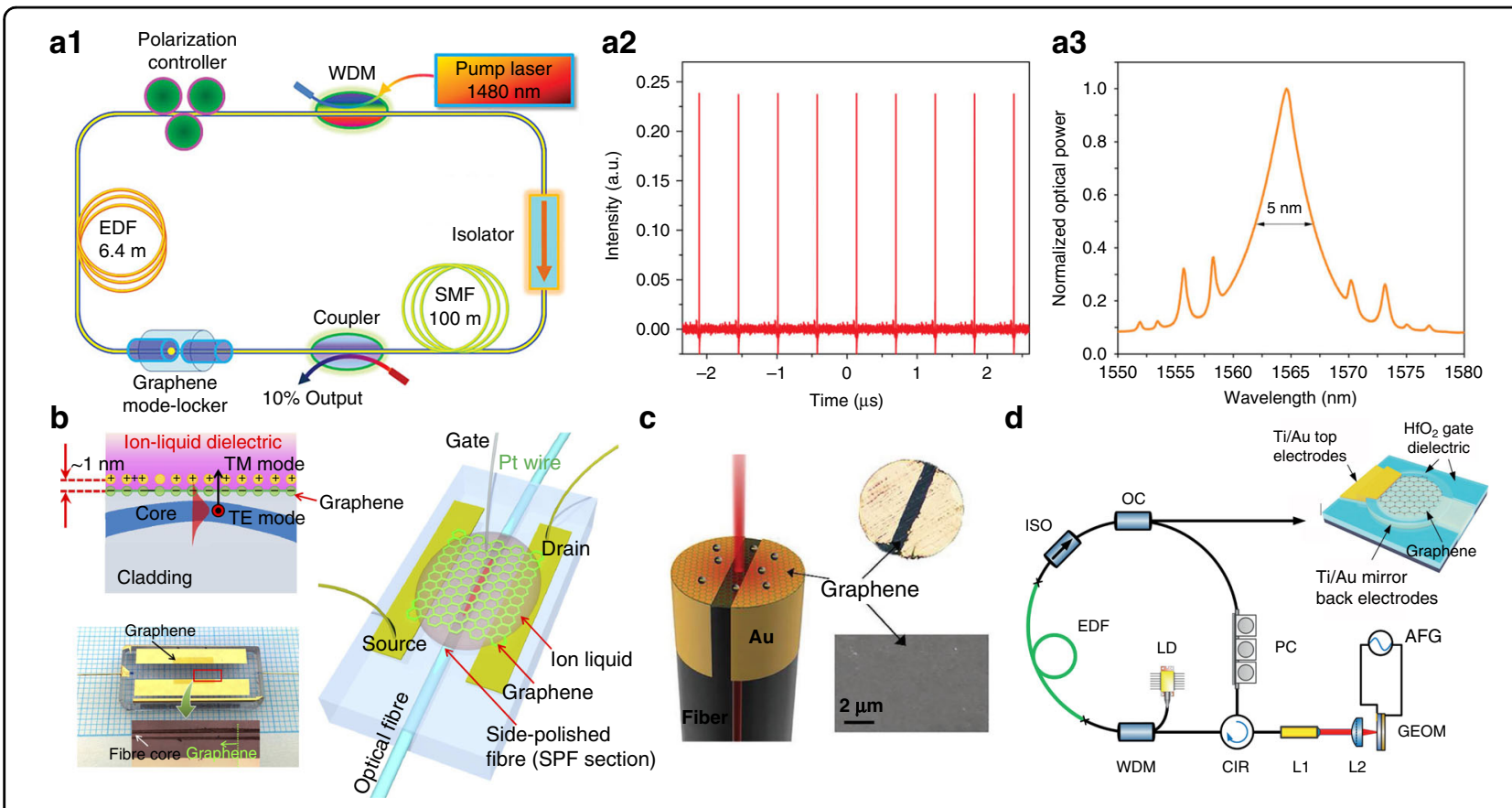

Fig. 8 Tunable graphene-based saturable absorber for mode-locked fiber laser. Laser configuration constituting a ring cavity (a1), typical laser output pulse train (a2), output pulse spectra centered at $1567 \mathrm{~nm}$ with solitonic sidebands (a3) ${ }^{44}$. Tunable graphene saturable absorbers by electrostatic gating $(\mathbf{b})^{96}$ and electric current effect $(\mathbf{c})^{94}$. d Actively mode-locked laser with a graphene-based EOM ${ }^{272}$. a1-a3 From ref. ${ }^{44}$. Copyright 2009, Wiley-VCH. b From ref. ${ }^{96}$. Reprinted with permission from Springer Nature: Nature Communications. c Reproduced from ref. ${ }^{94}$ with permission by Wiley-VCH, Copyright 2017. d From ref. ${ }^{272}$ Copyright 2018, Wiley-VCH

locked ultrafast laser by simply depositing graphene on a fiber endface, as shown in Fig. 8a1-a3. Compared with conventional semiconductor SA mirrors (SESAMs) and nanotubes, graphene $\mathrm{SA}$ is found to have an intrinsic wideband operation ${ }^{175}$. Moreover, the linear Dirac-cone electronic bandstructure allows the tunable saturable absorption by either electric gating or thermal effect ${ }^{95}$, thus the pulsed laser state can be actively controlled. Lee et al. ${ }^{96}$ first reported electro-static gating in graphene-DSF devices (Fig. 8b), and they realized electrically tunable fiber laser at various operational regimes. Later, Li et al. ${ }^{94}$ demonstrated state-variable fiber laser by engineering the Fermi-Dirac distribution of graphene based on an electric heating method (Fig. 8c). Recently, Bogusławski et al. ${ }^{272}$ adapted graphene-based EOM into a fiber cavity (Fig. 8d), and obtained electrically controlled repetition rate of generated pulses.

The integrations of 2D materials in fiber waveguide structures $^{54,56,180,182,273,274}$ are effective methods to enhance and manipulate the nonlinear optical interactions. Wu et al. ${ }^{186,275}$ reported cascaded four-wavemixing with graphene-coated-MF structure because of graphene's ultrahigh third-order nonlinearity. Chen et al. ${ }^{182}$ systematically studied the anisotropic response of SHG in hybrid $\mathrm{WS}_{2}-\mathrm{MF}$ as shown in Fig. 9a1-a2; furthermore, they demonstrated dynamic control of SHG by strain gauge (Fig. 9a3). Jiang et al. ${ }^{274}$ reported highefficiency second-order nonlinear processes (SHG and sum frequency generation) in an MF assisted by few-layer GaSe as shown in Fig. 9b1-b5. Fundamentally, 2Dmaterials enhanced optical nonlinearity is limited by the trade-off between absorption and interaction length. The defects absorption/scattering introduced during 2D materials transfer processes are always serious issues in current optical devices. Recently, Zuo et al. ${ }^{196}$ reported high crystalline as-grown $\mathrm{MoS}_{2}$ in 25-cm long HCF, and they observed that both SHG and third-harmonic generation (THG) were enhanced by 300 times compared with monolayer $\mathrm{MoS}_{2} /$ silica. This work will inspire development of clean 2D-materials-fiber devices with great potential of mass production and stimulate versatile nonlinear applications. Besides using their intrinsically high optical nonlinearity, the electrically tunable nonlinear response ${ }^{97,98,132}$ and plasmonic-enhanced nonlinearity in nanostructured 2D materials ${ }^{276,277}$ are promising directions for reconfigurable nonlinear fiber devices, which is difficult to achieve with conventional bulk materials.

\section{Challenges and opportunities}

In the past years, the silica optical fibers merged with $2 \mathrm{D}$ materials have stimulated rapid progresses for in-line 


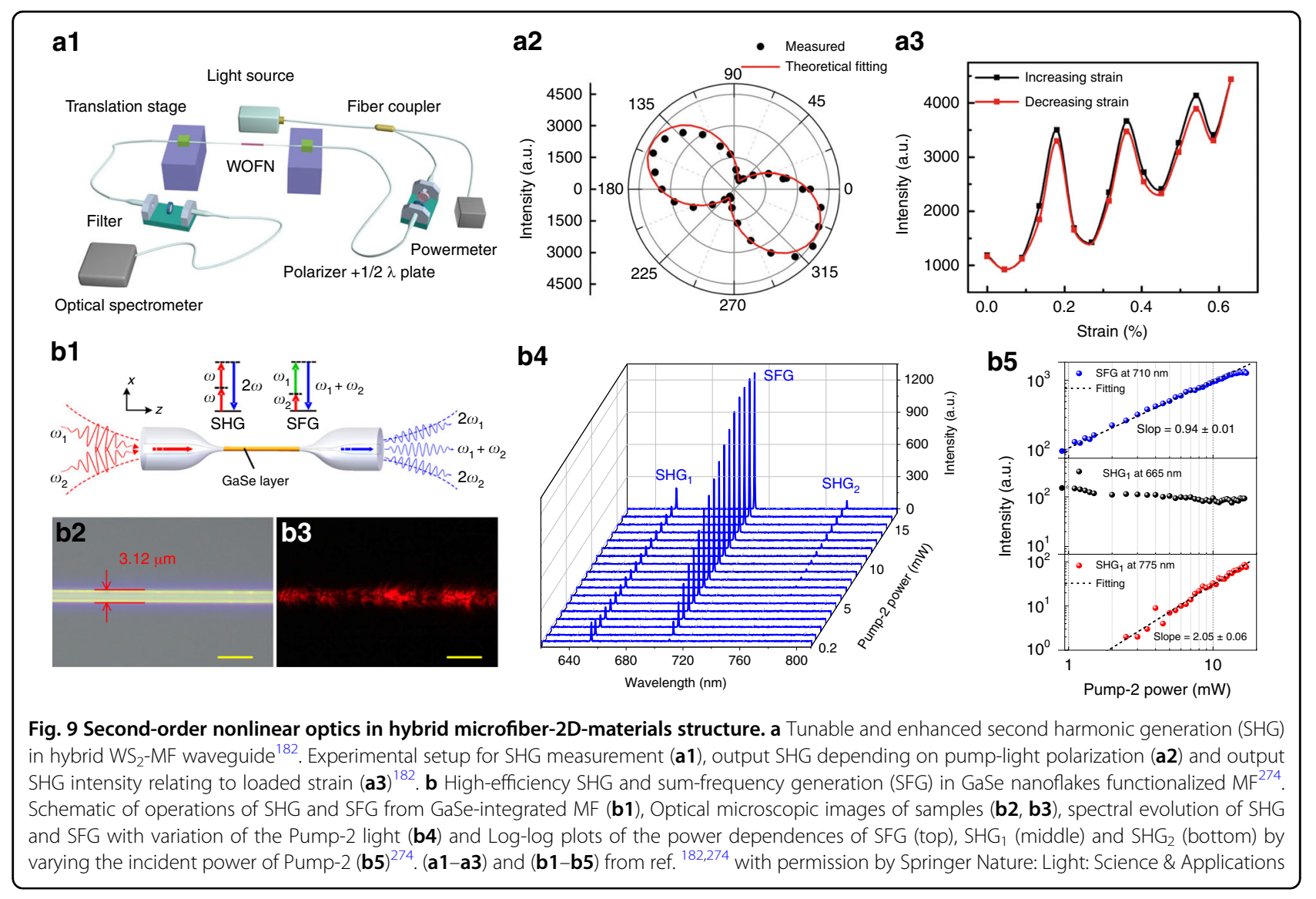

manipulating light beams in respect of polarization, phase, intensity and frequency, which shed light on the integrated all-fiber photonic and optoelectronic systems. Note that most of the applications remain at proof-of-concept or prototype stages, and many key challenges, such as batch reproduction of devices and reliable packaging are still waiting to be addressed towards the ultimate practical applications. With advanced fiber manufacturing, there are sophisticated techniques to fabricate various bulk fiber structures such as DSF, PCF, HCF and cleaved fiber-endface. With respect to materials production, different forms of 2D materials from the solutionprocessed nanosheets to the CVD-grown large-size single crystals are already available ${ }^{68,278,279}$. Nevertheless, the conventional mechanical transfer of $2 \mathrm{D}$ materials to fiber structures are time consuming and not scalable, and the unintentional doping and structural defects are easily introduced to hybrid fiber devices, which will significantly influence their optoelectronic performance $^{46,56,182,185,192,235,280}$. The recently developed functional inks and prints of 2D materials ${ }^{281}$ are gaining momentum for mass production with a high speed, low cost and moderate resolution $(<100 \mu \mathrm{m})$, and is potential for fabricating fiber devices that does not require crystallined 2D materials ${ }^{8,252,282}$. Using a direct CVD growth method to produce polycrystalline structure of 2D materials (graphene and $\mathrm{MoS}_{2}$ ) in PCF/HCF has also been achieved in 2019 ${ }^{195,196}$, which may provide the ultimate solution to most of the hybrid fiber devices. Device packaging is another vital issue to realize longterm and stable operation of $2 \mathrm{D}$ materials especially for air unstable materials, such as BP, $\mathrm{MoTe}_{2}$ and $\mathrm{Bi}_{2} \mathrm{Se}_{3}{ }^{145,283,284}$. The excellent electrical insulation, high thermal stability and chemical inertness render hexagonal boron nitride as one of the most important candidates for passivation and protection layer ${ }^{285}$.

As for the future development of hybrid fiber devices, the novel materials and advanced structures are two important ingredients for photonic and optoelectronic integration. Beyond the conventional graphene, BP and TMDCs, lots of other layered and non-layered 2D materials are discovered with diverging properties ${ }^{70,71,233,286}$, and their Van der Waals heterostructures further reveal unusual physics and properties ${ }^{66,67,287,288}$. In particular, the 2D magnets such as $\mathrm{CrI}_{3}$ and $\mathrm{Fe}_{3} \mathrm{GeTe}_{2}$ with magneto-optical Kerr effect show great potential for optical non-reciprocal fiber-devices at room temperature ${ }^{289,290}$. The twisted 2D materials with Moire pattern in graphene and TMDCs demonstrate exotic optical and electronic properties, which is intriguing to extend the 
optical spectra of photodetection and light emitting ${ }^{288,291}$ in fibers. In the scope of photonic structures, the interaction of confined chiral field in $\mathrm{MF}^{191}$ and valley polarization in TMDCs ${ }^{117-119}$, such as $\mathrm{MoS}_{2}$ and $\mathrm{WS}_{2}$, may promise novel all-fiber optical routers ${ }^{122}$. The BP of highly anisotropic linear and nonlinear optical response interacts the vector field in optical fiber modes should create extraordinary polarimetric fiber devices. On the other, the adaptation of external nanophotonic structures in DSF and fiber endface combining with 2D materials will achieve ultimate all-fiber light-matter interactions and light beam manipulation ${ }^{177}$. Beyond the classical-optic applications, the subwavelength MF is an efficient interface for coherent transfer of quantum states between atomic and photonic qubits, due to its strong transverse confinement of the guided field and the long interaction length along the propagation ${ }^{212}$. The emergence of $2 \mathrm{D}$ materials based SPEs in MF platform may provide an alternative fiber-integrated source and open promising new avenues for quantum photonics.

\section{Acknowledgements}

This work was sponsored by National Natural Science Foundation of China (62005231, 61535005 and 61925502), the National Science and Technology Major Projects (2017YFA0303700 and 2017YFC1403803), and Fundamental Research Funds for the Central Universities (20720200074).

\section{Conflict of interest}

The authors declare no conflict of interest.

Received: 19 November 2020 Revised: 14 March 2021 Accepted: 29 March 2021

Published online: 14 April 2021

\section{References}

1. Bao, X. Y. \& Chen, L. Recent progress in distributed fiber optic sensors. Sensors 12, 8601-8639 (2012).

2. Wang, B. Z. et al. High spatial resolution: an integrative review of its developments on the Brillouin optical time-and correlation-domain analysis. Meas. Sci. Technol. 31, 052001 (2020).

3. Plöschner, M., Tyc, T. \& Čižmár, T. Seeing through chaos in multimode fibres. Nat. Photonics 9, 529-535 (2015).

4. Qiu, L. et al. Multispectral light scattering endoscopic imaging of esophageal precancer. Light Sci. Appl. 7, 17174 (2018).

5. Gora, M. J. et al. Endoscopic optical coherence tomography: technologies and clinical applications [Invited]. Biomed. Opt. Express 8, 2405-2444 (2017).

6. Constable, A. et al. Demonstration of a fiber-optical light-force trap. Opt. Lett. 18, 1867-1869 (1993).

7. Liberale, C. et al. Miniaturized all-fibre probe for three-dimensional optical trapping and manipulation. Nat. Photonics 1, 723-727 (2007).

8. Shi, W. et al. Fiber lasers and their applications [Invited]. Appl. Opt. 53, 6554-6568 (2014).

9. Zervas, M. N. \& Codemard, C. A. High power fiber lasers: a review. IEEE J. Sel. Top. Quantum Electron. 20, 0904123 (2014).

10. Agrawal, G. P. Nonlinear Fiber Optics. (Boston, Academic Press, 1989)

11. Hill, K. O. \& Meltz, G. Fiber Bragg grating technology fundamentals and overview. J. Lightwave Technol. 15, 1263-1276 (1997).

12. Rao, Y. J. In-fibre Bragg grating sensors. Meas. Sci. Technol. 8, 355-375 (1997).

13. Albert, J., Shao, L. Y. \& Caucheteur, C. Tilted fiber Bragg grating sensors. Laser Photonics Rev. 7, 83-108 (2013).
14. Wang, Y. P. Review of long period fiber gratings written by $\mathrm{CO}_{2}$ laser. J. Appl. Phys. 108, 081101 (2010).

15. Yeh, P., Yariv, A. \& Marom, E. Theory of Bragg fiber. J. Optical Soc. Am. 68 , 1196-1201 (1978).

16. Knight, J. C. et al. Photonic band gap guidance in optical fibers. Science $\mathbf{2 8 2}$, 1476-1478 (1998)

17. Cregan, R. F. et al. Single-mode photonic band gap guidance of light in air. Science 285, 1537-1539 (1999).

18. Russell, P. Photonic crystal fibers. Science 299, 358-362 (2003).

19. Tong, L. M. et al. Subwavelength-diameter silica wires for low-loss optical wave guiding. Nature 426, 816-819 (2003).

20. Knight, J. C. et al. Phase-matched excitation of whispering-gallery-mode resonances by a fiber taper. Opt. Lett. 22, 1129-1131 (1997).

21. Brambilla, G. et al. Optical fiber nanowires and microwires: fabrication and applications. Adv. Opt. Photonics 1, 107-161 (2009).

22. Zhang, L., Tang, Y. \& Tong, L. M. Micro-/nanofiber optics: merging photonics and material science on nanoscale for advanced sensing technology. iscience 23, 100810 (2020)

23. Tong, L. M. et al. Optical microfibers and nanofibers: a tutorial. Opt. Commun. 285, 4641-4647 (2012)

24. Chen, J. H., Li, D. R. \& Xu, F. Optical microfiber sensors: sensing mechanisms, and recent advances. J. Lightwave Technol. 37, 2577-2589 (2019).

25. Wang, P., Wang, Y. P. \& Tong, L. M. Functionalized polymer nanofibers: a versatile platform for manipulating light at the nanoscale. Light Sci. Appl. 2, e102 (2013).

26. Abouraddy, A. F. et al. Towards multimaterial multifunctional fibres that see, hear, sense and communicate. Nat. Mater. 6, 336-347 (2007).

27. Temelkuran, B. et al. Wavelength-scalable hollow optical fibres with large photonic bandgaps for $\mathrm{CO}_{2}$ laser transmission. Nature 420, 650-653 (2002).

28. Canales, A. et al. Multifunctional fibers for simultaneous optical, electrical and chemical interrogation of neural circuits in vivo. Nat. Biotechnol. 33, 277-284 (2015).

29. Schmidt, M. A., Argyros, A. \& Sorin, F. Hybrid optical fibers-an innovative platform for in-fiber photonic devices. Adv. Optical Mater. 4, 13-36 (2016).

30. Novoselov, K. S. et al. Electric field effect in atomically thin carbon films. Science 306, 666-669 (2004).

31. Geim, A. K. \& Novoselov, K. S. The rise of graphene. Nat. Mater. 6, 183-191 (2007).

32. Bonaccorso, F. et al. Graphene photonics and optoelectronics. Nat. Photonics 4, 611-622 (2010).

33. Bonaccorso, F. et al. Graphene, related two-dimensional crystals, and hybrid systems for energy conversion and storage. Science 347, 1246501 (2015).

34. Weiss, N. O. et al. Graphene: an emerging electronic material. Adv. Mater. 24, 5782-5825 (2012).

35. Butler, S. Z. et al. Progress, challenges, and opportunities in two-dimensional materials beyond graphene. ACS Nano 7, 2898-2926 (2013).

36. Novoselov, K. S. et al. A roadmap for graphene. Nature 490, 192-200 (2012).

37. Wang, Y. X. et al. Thermal properties of two dimensional layered materials. Adv. Funct. Mater. 27, 1604134 (2017).

38. Bao, Q. L. \& Loh, K. P. Graphene photonics, plasmonics, and broadband optoelectronic devices. ACS Nano 6, 3677-3694 (2012).

39. Tan, T. et al. 2D Material optoelectronics for information functional device applications: status and challenges. Adv. Sci. 7, 2000058 (2020).

40. Youngblood, N. \& Li, M. Integration of $2 \mathrm{D}$ materials on a silicon photonics platform for optoelectronics applications. Nanophotonics 6, 1205-1218 (2017)

41. Ferrari, A. C. et al. Science and technology roadmap for graphene, related two-dimensional crystals, and hybrid systems. Nanoscale 7, 4598-4810 (2015).

42. Yu, S. L. et al. 2D materials for optical modulation: challenges and opportunities. Adv. Mater. 29, 1606128 (2017).

43. Sun, Z. P., Martinez, A. \& Wang, F. Optical modulators with 2D layered materials. Nat. Photonics 10, 227-238 (2016).

44. Bao, Q. L. et al. Atomic-layer graphene as a saturable absorber for ultrafast pulsed lasers. Adv. Funct. Mater. 19, 3077-3083 (2009).

45. Sun, Z. P. et al. Graphene mode-locked ultrafast laser. ACS Nano 4, 803-810 (2010)

46. Bao, Q. L. et al. Broadband graphene polarizer. Nat. Photonics 5, 411-415 (2011).

47. Kou, J. L. et al. Platform for enhanced light-graphene interaction length and miniaturizing fiber stereo devices. Optica 1, 307-310 (2014). 
48. Wu, Y. et al. Graphene-coated microfiber Bragg grating for high-sensitivity gas sensing. Opt. Lett. 39, 1235-1237 (2014).

49. Yao, B. C. et al. Graphene enhanced evanescent field in microfiber multimode interferometer for highly sensitive gas sensing. Opt. Express 22 28154-28162 (2014).

50. $\mathrm{Ma}$, J. et al. High-sensitivity fiber-tip pressure sensor with graphene diaphragm. Opt. Lett. 37, 2493-2495 (2012).

51. Zheng, B. C. et al. Miniature optical fiber current sensor based on a graphene membrane. Laser Photonics Rev. 9, 517-522 (2015).

52. Yan, S. C. et al. Optical electrical current sensor utilizing a graphenemicrofiber-integrated coil resonator. Appl. Phys. Lett. 107, 053502 (2015).

53. Cao, Z. X. et al. Biochemical sensing in graphene-enhanced microfiber resonators with individual molecule sensitivity and selectivity. Light Sci. Appl. 8, 107 (2019).

54. An, N. et al. Electrically tunable four-wave-mixing in graphene heterogeneous fiber for individual gas molecule detection. Nano Lett. 20, 6473-6480 (2020).

55. Liu, Z. B. et al. Broadband all-optical modulation using a graphene-coveredmicrofiber. Laser Phys. Lett. 10, 065901 (2013).

56. Li, W. et al. Ultrafast all-optical graphene modulator. Nano Lett. 14, 955-959 (2014).

57. Gan, X. T. et al. Graphene-assisted all-fiber phase shifter and switching. Optica 2, 468-471 (2015).

58. Chen, J. H. et al. An all-optical modulator based on a stereo graphene-microfiber structure. Light Sci. Appl. 4, e360 (2015).

59. Mak, K. F. \& Shan, J. Photonics and optoelectronics of 2D semiconductor transition metal dichalcogenides. Nat. Photonics 10, 216-226 (2016).

60. Kao, K. C. \& Hockham, G. A. Dielectric-fibre surface waveguides for optical frequencies. Proc. Inst. Electr. Eng. 113, 1151-1158 (1966).

61. Nagayama, K. et al. Ultra-low-loss $(0.1484 \mathrm{~dB} / \mathrm{km})$ pure silica core fibre and extension of transmission distance. Electron. Lett. 38, 1168-1169 (2002).

62. Musgraves J. D., Hu, J. J. \& Calvez, L. Springer Handbook of Glass. (Nature Switzerland AG, Springer, 2019)

63. Lægsgaard, J. Theory of surface second-harmonic generation in silica nanowires. J. Optical Soc. Am. B 27, 1317-1324 (2010).

64. Guyot-Sionnest, P. \& Shen, Y. R. Bulk contribution in surface second-harmonic generation. Phys. Rev. B Condens. Matter 38, 7985-7989 (1988).

65. Brambilla, G. \& Payne, D. N. The ultimate strength of glass silica nanowires. Nano Lett. 9, 831-835 (2009).

66. Novoselov, K. S. et al. 2D materials and van der Waals heterostructures Science 353, aac9439 (2016)

67. Duong, D. L., Yun, S. J. \& Lee, Y. H. van der Waals layered materials: opportunities and challenges. ACS Nano 11, 11803-11830 (2017).

68. Zhou, J. D. et al. A librany of atomically thin metal chalcogenides. Nature $\mathbf{5 5 6}$ 355-359 (2018).

69. Zhang, H. Ultrathin two-dimensional nanomaterials. ACS Nano 9, 9451-9469 (2015).

70. Glavin, N. R. et al. Emerging applications of elemental 2D materials. Adv. Mater. 32, 1904302 (2020).

71. Ashton, M. et al. Topology-scaling identification of layered solids and stable exfoliated 2D materials. Phys. Rev. Lett. 118, 106101 (2017).

72. Cao, Y. et al. Unconventional superconductivity in magic-angle graphene superlattices. Nature 556, 43-50 (2018)

73. Cao, Y. et al. Correlated insulator behaviour at half-filling in magic-angle graphene superlattices. Nature 556, 80-84 (2018).

74. Dresselhaus, M. S., Dresselhaus, G. \& Eklund, P. C. Science of Fullerenes and Carbon Nanotubes: Their Properties and Applications. (San Diego, Academic Press, 1996)

75. Castro, E. V. et al. Limits on charge carrier mobility in suspended graphene due to flexural phonons. Phys. Rev. Lett. 105, 266601 (2010).

76. $\mathrm{Wu}, \mathrm{Y}$. Q. et al. High-frequency, scaled graphene transistors on diamond-like carbon. Nature 472, 74-78 (2011)

77. Lin, Y. M. et al. $100-\mathrm{GHz}$ transistors from wafer-scale epitaxial graphene. Science 327, 662 (2010).

78. Hanson, G. W. Dyadic Green's functions and guided surface waves for a surface conductivity model of graphene. J. Appl. Phys. 103, 064302 (2008).

79. Hao, T., Chang, Z. S. \& Chiang, K. S. Comparison of different optical models of graphene for the analysis of graphene-attached microfibers and D-shaped fibers. Opt. Commun. 452, 347-354 (2019).

80. Nair, R. R. et al. Fine structure constant defines visual transparency of graphene. Science 320, 1308 (2008).
81. Wang, F. et al. Gate-variable optical transitions in graphene. Science $\mathbf{3 2 0}$ 206-209 (2008).

82. Dawlaty, J. M. et al. Measurement of ultrafast carrier dynamics in epitaxial graphene. Appl. Phys. Lett. 92, 042116 (2008).

83. Sun, D. et al. Ultrafast relaxation of excited dirac fermions in epitaxial graphene using optical differential transmission spectroscopy. Phys. Rev. Lett. 101, 157402 (2008).

84. Gusynin, V. P., Sharapov, S. G. \& Carbotte, J. P. Magneto-optical conductivity in graphene. J. Phys. Condens. Matter 19, 026222 (2006).

85. Pellegrino, F. M. D., Angilella, G. G. N. \& Pucci, R. Strain effect on the optical conductivity of graphene. Phys. Rev. B Condens. Matter 81, 035411 (2010).

86. $\mathrm{Ni}, \mathrm{G}$. X. et al. Tuning optical conductivity of large-scale CVD graphene by strain engineering. Adv. Mater. 26, 1081-1083 (2014).

87. Schedin, F. et al. Detection of individual gas molecules adsorbed on graphene. Nat. Mater. 6, 652-655 (2007).

88. Shivananju, B. N. et al. The roadmap of graphene-based optical biochemical sensors. Adv. Funct. Mater. 27, 1603918 (2017).

89. Hendry, E. et al. Coherent nonlinear optical response of graphene. Phys. Rev. Lett. 105, 097401 (2010).

90. Säynätjoki, A. et al. Rapid large-area multiphoton microscopy for characterization of graphene. ACS Nano 7, 8441-8446 (2013).

91. Cheng, J. L., Vermeulen, N. \& Sipe, J. E. Third order optical nonlinearity of graphene. N. J. Phys. 16, 053014 (2014).

92. $\mathrm{Ma}$, C. Y. et al. Recent progress in ultrafast lasers based on $2 \mathrm{D}$ materials as a saturable absorber. Appl. Phys. Rev. 6, 041304 (2019).

93. Liu, W. J. et al. Recent advances of $2 \mathrm{D}$ materials in nonlinear photonics and fiber Lasers. Adv. Optical Mater. 8, 1901631 (2020).

94. Li, C. et al. Manipulation of nonlinear optical properties of graphene bonded fiber devices by thermally engineering fermi-dirac distribution. Adv. Optical Mater. 5, 1700630 (2017).

95. Marini, A., Cox, J. D. \& de Abajo, F. J. G. Theory of graphene saturable absorption. Phys. Rev. B 95, 125408 (2017).

96. Lee, E. J. et al. Active control of all-fibre graphene devices with electrical gating. Nat. Commun. 6, 6851 (2015).

97. Jiang, T. et al. Gate-tunable third-order nonlinear optical response of massless Dirac fermions in graphene. Nat. Photonics 12, 430-436 (2018).

98. Soavi, G. et al. Broadband, electrically tunable third-harmonic generation in graphene. Nat. Nanotechnol. 13, 583-588 (2018).

99. Glazov, M. M. \& Ganichev, S. D. High frequency electric field induced nonlinear effects in graphene. Phys. Rep. 535, 101-138 (2014).

100. Yoshikawa, N., Tamaya, T. \& Tanaka, K. High-harmonic generation in graphene enhanced by elliptically polarized light excitation. Science $\mathbf{3 5 6}$ 736-738 (2017).

101. Hafez, H. A. et al. Extremely efficient terahertz high-harmonic generation in graphene by hot Dirac fermions. Nature 561, 507-511 (2018).

102. Liu, L. et al. Graphene oxidation: thickness-dependent etching and strong chemical doping. Nano Lett. 8, 1965-1970 (2008).

103. Lee, C. et al. Measurement of the elastic properties and intrinsic strength of monolayer graphene. Science 321, 385-388 (2008).

104. Kim, K. S. et al. Large-scale pattern growth of graphene films for stretchable transparent electrodes. Nature 457, 706-710 (2009).

105. Wang, Q. H. et al. Electronics and optoelectronics of two-dimensional transition metal dichalcogenides. Nat. Nanotechnol. 7, 699-712 (2012).

106. Jariwala, D. et al. Emerging device applications for semiconducting twodimensional transition metal dichalcogenides. ACS Nano 8, 1102-1120 (2014).

107. Manzeli, S. et al. 2D transition metal dichalcogenides. Nat. Rev. Mater. 2, 17033 (2017).

108. Splendiani, A. et al. Emerging photoluminescence in monolayer MoS 2. Nano Lett. 10, 1271-1275 (2010).

109. Mak, K. F. et al. Atomically thin $\mathrm{MoS}_{2}$ : a new direct-gap semiconductor. Phys. Rev. Lett. 105, 136805 (2010).

110. Hill, H. M. et al. Observation of excitonic Rydberg states in monolayer $\mathrm{MoS}_{2}$ and $W S_{2}$ by photoluminescence excitation spectroscopy. Nano Lett. $\mathbf{1 5}$ 2992-2997 (2015).

111. Mak, K. F. et al. Tightly bound trions in monolayer MoS2. Nat. Mater. 12 207-211 (2013).

112. Wang, G. et al. Colloquium: excitons in atomically thin transition metal dichalcogenides. Rev. Mod. Phys. 90, 021001 (2018).

113. Chernikov, A. et al. Electrical tuning of exciton binding energies in monolayer WS 2 . Phys. Rev. Lett. 115, 126802 (2015). 
114. Wang, Y. L. et al. Strain-induced direct-indirect bandgap transition and phonon modulation in monolayer WS . Nano Res. 8, 2562-2572 (2015).

115. Liu, H. L. et al. Optical properties of monolayer transition metal dichalcogenides probed by spectroscopic ellipsometry. Appl. Phys. Lett. 105, 201905 (2014).

116. Li, Y. L. et al. Measurement of the optical dielectric function of monolayer transition-metal dichalcogenides: $\mathrm{MoS}_{2}, \mathrm{MoSe}_{2}, \mathrm{WS}_{2}$ and WSe $\mathrm{Wh}_{2}$. Phys. Rev. $B$ 90, 205422 (2014)

117. Xiao, D. et al. Coupled spin and valley physics in monolayers of MoS and other group-VI dichalcogenides. Phys. Rev. Lett. 108, 196802 (2012).

118. Mak, K. F. et al. Control of valley polarization in monolayer $\mathrm{MoS}_{2}$ by optical helicity. Nat. Nanotechnol. 7, 494-498 (2012).

119. Zeng, H. L. et al. Valley polarization in $\mathrm{MoS}_{2}$ monolayers by optical pumping Nat. Nanotechnol. 7, 490-493 (2012).

120. Sun, L. Y. et al. Separation of valley excitons in a MoS 2 monolayer using a subwavelength asymmetric groove array. Nat. Photonics 13, 180-184 (2019).

121. Wang, J. J. et al. Routing valley exciton emission of a $W_{2}$ monolayer via delocalized Bloch modes of in-plane inversion-symmetry-broken photonic crystal slabs. Light Sci. Appl. 9, 148 (2020).

122. Gong, S. H. et al. Nanoscale chiral valley-photon interface through optical spin-orbit coupling. Science 359, 443-447 (2018).

123. Sun, J. W. et al. Selectively depopulating valley-polarized excitons in monolayer $\mathrm{MoS}_{2}$ by local chirality in single plasmonic nanocavity. Nano Lett. 20, 4953-4959 (2020).

124. Li, Z. W. et al. Tailoring $\mathrm{MoS}_{2}$ valley-polarized photoluminescence with super chiral near-field. Adv. Mater. 30, 1801908 (2018)

125. Kumar, N. et al. Second harmonic microscopy of monolayer $\mathrm{MoS}_{2}$. Phys. Rev. B Condens. Matter 87, 161403 (2013).

126. Malard, L. M. et al. Observation of intense second harmonic generation from $\mathrm{MoS}_{2}$ atomic crystals. Phys. Rev. B Condens. Matter 87, 201401(R) (2013).

127. Li, Y. L. et al. Probing symmetry properties of few-layer $\mathrm{MoS}_{2}$ and h-BN by optical second-harmonic generation. Nano Lett. 13, 3329-3333 (2013).

128. Hsu, W. T. et al. Second harmonic generation from artificially stacked transition metal dichalcogenide twisted bilayers. ACS Nano 8, 2951-2958 (2014).

129. Zhao, M. et al. Atomically phase-matched second-harmonic generation in a 2D crystal. Light Sci. Appl. 5, e16131 (2016).

130. Janisch, C. et al. Extraordinary Second Harmonic Generation in tungsten disulfide monolayers. Sci. Rep. 4, 5530 (2014).

131. Yin, X. B. et al. Edge nonlinear optics on a $\mathrm{MoS}_{2}$ atomic monolayer. Science 344, 488-490 (2014)

132. Seyler, K. L. et al. Electrical control of second-harmonic generation in a WSe monolayer transistor. Nat. Nanotechnol. 10, 407-411 (2015).

133. Wang, R. et al. Third-harmonic generation in ultrathin films of $\mathrm{MOS}_{2}$. ACS Appl. Mater. Interfaces 6, 314-318 (2014).

134. Wang, K. P. et al. Ultrafast saturable absorption of two-dimensional MoS $_{2}$ nanosheets. ACS Nano 7, 9260-9267 (2013).

135. Wang, S. X. et al. Broadband few-layer $\mathrm{MoS}_{2}$ saturable absorbers. Adv. Mater. 26, 3538-3544 (2014)

136. Li, Y. X. et al. Giant two-photon absorption in monolayer $\mathrm{MoS}_{2}$. Laser Photonics Rev. 9, 427-434 (2015).

137. Bertolazzi, S., Brivio, J. \& Kis, A. Stretching and breaking of ultrathin MoS2. ACS Nano 5, 9703-9709 (2011).

138. Akinwande, D. et al. A review on mechanics and mechanical properties of $2 \mathrm{D}$ materials - Graphene and beyond. Extrem. Mech. Lett. 13, $42-77$ (2017).

139. Zhou, Y. \& Reed, E. J. Structural phase stability control of monolayer MoTe with adsorbed atoms and molecules. J. Phys. Chem. C. 119, 21674-21680 (2015).

140. Duerloo, K. A. N., Li, Y. \& Reed, E. J. Structural phase transitions in twodimensional Mo- and W-dichalcogenide monolayers. Nat. Commun. 5, 4214 (2014).

141. Song, S. et al. Room temperature semiconductor-metal transition of MoTe thin films engineered by strain. Nano Lett. 16, 188-193 (2016).

142. Li, Y. et al. Structural semiconductor-to-semimetal phase transition in twodimensional materials induced by electrostatic gating. Nat. Commun. 7, 10671 (2016).

143. Wang, Y. et al. Structural phase transition in monolayer $\mathrm{MoTe}_{2}$ driven by electrostatic doping. Nature 550, 487-491 (2017).

144. Li, H. K. et al. Valley optomechanics in a monolayer semiconductor. Nat. Photonics 13, 397-401 (2019).
145. Xia, F. N., Wang, H. \& Jia, Y. C. Rediscovering black phosphorus as an anisotropic layered material for optoelectronics and electronics. Nat. Commun. 5, 4458 (2014)

146. Liu, H. et al. Phosphorene: an unexplored 2D semiconductor with a high hole mobility. ACS Nano 8, 4033-4041 (2014).

147. Koenig, S. P. et al. Electric field effect in ultrathin black phosphorus. Appl. Phys. Lett. 104, 103106 (2014).

148. Li, L. K. et al. Black phosphorus field-effect transistors. Nat. Nanotechnol. 9 372-377 (2014).

149. Yang, J. et al. Optical tuning of exciton and trion emissions in monolayer phosphorene. Light Sci. Appl. 4, e312 (2015).

150. Zhang, S. et al. Extraordinary photoluminescence and strong temperature/ angle-dependent raman responses in few-layer phosphorene. ACS Nano $\mathbf{8}$ 9590-9596 (2014).

151. Li, L. K. et al. Direct observation of the layer-dependent electronic structure in phosphorene. Nat. Nanotechnol. 12, 21-25 (2017).

152. Low, T. et al. Tunable optical properties of multilayer black phosphorus thin films. Phys. Rev. B 90, 075434 (2014).

153. Tran, V. et al. Layer-controlled band gap and anisotropic excitons in few-layer black phosphorus. Phys. Rev. B 89, 235319 (2014).

154. Yuan, H. T. et al. Polarization-sensitive broadband photodetector using a black phosphorus vertical p-n junction. Nat. Nanotechnol. 10, 707-713 (2015).

155. Engel, M., Steiner, M. \& Avouris, P. Black phosphorus photodetector for multispectral, high-resolution imaging. Nano Lett. 14, 6414-6417 (2014).

156. Buscema, M. et al. Photovoltaic effect in few-layer black phosphorus PN junctions defined by local electrostatic gating. Nat. Commun. 5, 4651 (2014).

157. Chen, C. et al. Bright mid-infrared photoluminescence from thin-film black phosphorus. Nano Lett. 19, 1488-1493 (2019).

158. Jiang, J. W. \& Park, H. S. Mechanical properties of single-layer black phosphorus. J. Phys. D Appl. Phys. 47, 385304 (2014)

159. Wei, Q. \& Peng, X. H. Superior mechanical flexibility of phosphorene and fewlayer black phosphorus. Appl. Phys. Lett. 104, 251915 (2014).

160. Qiao, J. S. et al. High-mobility transport anisotropy and linear dichroism in few-layer black phosphorus. Nat. Commun. 5, 4475 (2014).

161. Wang, X. M. et al. Highly anisotropic and robust excitons in monolayer black phosphorus. Nat. Nanotechnol. 10, 517-521 (2015).

162. Wang, X. M. \& Lan, S. F. Optical properties of black phosphorus. Adv. Opt. Photonics 8, 618-655 (2016).

163. Chen, Y. et al. Mechanically exfoliated black phosphorus as a new saturable absorber for both Q-switching and Mode-locking laser operation. Opt. Express 23, 12823-12833 (2015).

164. Lu, S. B. et al. Broadband nonlinear optical response in multi-layer black phosphorus: an emerging infrared and mid-infrared optical material. Opt Express 23, 11183-11194 (2015).

165. Zheng, J. L. et al. Black phosphorus based all-optical-signal-processing: toward high performances and enhanced stability. ACS Photonics 4, 1466-1476 (2017).

166. Uddin, S. et al. Nonlinear black phosphorus for ultrafast optical switching. Sci. Rep. 7, 43371 (2017)

167. Autere, A. et al. Rapid and large-area characterization of exfoliated black phosphorus using third-harmonic generation microscopy. J. Phys. Chem. Lett. 8, 1343-1350 (2017).

168. Youngblood, N. et al. Layer-tunable third-harmonic generation in multilayer black phosphorus. ACS Photonics 4, 8-14 (2017).

169. Tao, J. et al. Mechanical and electrical anisotropy of few-layer black phosphorus. ACS Nano 9, 11362-11370 (2015).

170. Quereda, J. et al. Strong modulation of optical properties in black phosphorus through strain-engineered rippling. Nano Lett. 16, 2931-2937 (2016).

171. Fei, R. X. \& Yang, L. Strain-engineering the anisotropic electrical conductance of few-layer black phosphorus. Nano Lett. 14, 2884-2889 (2014).

172. Rodin, A. S., Carvalho, A. \& Neto, A. H. C. Strain-induced gap modification in black phosphorus. Phys. Rev. Lett. 112, 176801 (2014).

173. Çakır, D., Sahin, H. \& Peeters, F. M. Tuning of the electronic and optical properties of single-layer black phosphorus by strain. Phys. Rev. B 90, 205421 (2014).

174. Dhanabalan, S. C. et al. Emerging trends in phosphorene fabrication towards next generation devices. Adv. Sci. 4, 1600305 (2017).

175. Martinez, A. \& Sun, Z. P. Nanotube and graphene saturable absorbers for fibre lasers. Nat. Photonics 7, 842-845 (2013). 
176. Koenderink, A. F., Alù, A. \& Polman, A. Nanophotonics: shrinking light-based technology. Science 348, 516-521 (2015).

177. Vaiano, P. et al. Lab on fiber technology for biological sensing applications. Laser Photonics Rev. 10, 922-961 (2016).

178. Yu, F. F. et al. Ultrasensitive pressure detection of few-layer $\mathrm{MoS}_{2}$. Adv. Mater. 29, 1603266 (2017)

179. Ying, Y. et al. Recent research progress of optical fiber sensors based on Dshaped structure. Opt. Laser Technol. 90, 149-157 (2017).

180. Zhang, H. J. et al. Enhanced all-optical modulation in a graphene-coated fibre with low insertion loss. Sci. Rep. 6, 23512 (2016).

181. Luo, Y. H. et al. Tungsten disulfide $\left(\mathrm{WS}_{2}\right)$ based all-fiber-optic humidity sensor. Opt. Express 24, 8956-8966 (2016).

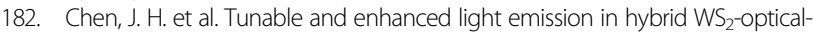
fiber-nanowire structures. Light.: Sci. Appl. 8, 8 (2019).

183. Li, C. et al. A fiber laser using graphene-integrated 3-D microfiber coil. IEEE Photonics J. 8, 1500307 (2016).

184. Chen, J. H. et al. Mechanical modulation of a hybrid graphene-microfiber structure. Adv. Optical Mater. 4, 853-857 (2016).

185. Luo, Z. C. et al. Microfiber-based few-layer black phosphorus saturable absorber for ultra-fast fiber laser. Opt. Express 23, 20030-20039 (2015).

186. Wu, Y. et al. Generation of cascaded four-wave-mixing with graphene-coated microfiber. Photonics Res. 3, A64-A68 (2015).

187. He, Y. M. et al. Single quantum emitters in monolayer semiconductors. Nat. Nanotechnol. 10, 497-502 (2015).

188. Koperski, M. et al. Single photon emitters in exfoliated WSe $\mathrm{S}_{2}$ structures. Nat. Nanotechnol. 10, 503-506 (2015).

189. Autere, A. et al. Nonlinear optics with 2D layered materials. Adv. Mater. 30, 1705963 (2018).

190. Wen, X. L., Gong, Z. B. \& Li, D. H. Nonlinear optics of two-dimensional transition metal dichalcogenides. InfoMat 1, 317-337 (2019).

191. Petersen, J., Volz, J. \& Rauschenbeutel, A. Chiral nanophotonic waveguide interface based on spin-orbit interaction of light. Science 346, 67-71 (2014).

192. Liao, F. et al. Enhancing monolayer photoluminescence on optical micro/ nanofibers for low-threshold lasing. Sci. Adv. 5, eaax7398 (2019).

193. Lodahl, P. et al. Chiral quantum optics. Nature 541, 473-480 (2017).

194. Markos, C. et al. Hybrid photonic-crystal fiber. Rev. Mod. Phys. 89, 045003 (2017).

195. Chen, K. et al. Graphene photonic crystal fibre with strong and tunable light-matter interaction. Nat. Photonics 13, 754-759 (2019).

196. Zuo, Y. G. et al. Optical fibres with embedded two-dimensional materials for ultrahigh nonlinearity. Nat. Nanotechnol. 15, 987-991 (2020).

197. Choi, S. Y. et al. Graphene-filled hollow optical fiber saturable absorber for efficient soliton fiber laser mode-locking. Opt. Express 20, 5652-5657 (2012).

198. Lin, Y. H. et al. Using graphene nano-particle embedded in photonic crystal fiber for evanescent wave mode-locking of fiber laser. Opt. Express 21, 16763-16776 (2013).

199. Huang, M. et al. Ultrasensitive sensing in air based on graphene-coated hollow core fibers. Opt. Express 26, 3098-3107 (2018).

200. Chen, J. H. et al. Multifunctional optical nanofiber polarization devices with 3D geometry. Opt. Express 22, 17890-17896 (2014).

201. de Oliveira, R. E. P. \& de Matos, C. J. S. Graphene based waveguide polarizers: in-depth physical analysis and relevant parameters. Sci. Rep. 5, 16949 (2015)

202. Kim, J. T. \& Choi, C. G. Graphene-based polymer waveguide polarizer. Opt. Express 20, 3556-3562 (2012).

203. Liu, M. et al. A graphene-based broadband optical modulator. Nature $\mathbf{4 7 4}$ 64-67 (2011)

204. Cui, Y. D., Lu, F. F. \& Liu, X. M. Nonlinear saturable and polarization-induced absorption of rhenium disulfide. Sci. Rep. 7, 40080 (2017).

205. Liu, E. F. et al. Integrated digital inverters based on two-dimensional anisotropic $\mathrm{ReS}_{2}$ field-effect transistors. Nat. Commun. 6, 6991 (2015).

206. Zheng, W. H. et al. Light emission properties of 2D transition metal dichalcogenides: fundamentals and applications. Adv. Optical Mater. 6, 1800420 (2018)

207. Wang, Z. W. et al. Two-dimensional light-emitting materials: preparation, properties and applications. Chem. Soc. Rev. 47, 6128-6174 (2018).

208. Tran, T. T. et al. Quantum emission from hexagonal boron nitride monolayers. Nat. Nanotechnol. 11, 37-41 (2016).

209. Toth, M. \& Aharonovich, I. Single photon sources in atomically thin materials. Annu. Rev. Phys. Chem. 70, 123-142 (2019).

210. Aharonovich, l., Englund, D. \& Toth, M. Solid-state single-photon emitters. Nat. Photonics 10, 631-641 (2016).
211. Schell, A. W. et al. Coupling quantum emitters in $2 D$ materials with tapered fibers. ACS Photonics 4, 761-767 (2017).

212. Nayak, K. P. et al. Nanofiber quantum photonics. J. Opt. 20, 073001 (2018).

213. Liu, J. M. et al. Review of graphene modulators from the low to the high figure of merits. J. Phys. D: Appl. Phys. 53, 233002 (2020).

214. Yu, S. L. et al. Graphene decorated microfiber for ultrafast optical modulation. Opt. Express 23, 10764-10770 (2015).

215. He, X. Y., Xu, M. \& Zhang, X. C. Theoretical investigation of a broadband alloptical graphene-microfiber modulator. J. Optical Soc. Am. B 33, 2588-2595 (2016).

216. Yu, S. L. et al. All-optical graphene modulator based on optical Kerr phase shift. Optica 3, 541-544 (2016).

217. Song, Y. F. et al. Nonlinear few-layer antimonene-based all-optical signal processing: ultrafast optical switching and high-speed wavelength conversion. Adv. Optical Mater. 6, 1701287 (2018).

218. Wang, Y. D. et al. All-optical control of microfiber resonator by graphene's photothermal effect. Appl. Phys. Lett. 108, 171905 (2016).

219. Wu, K. et al. All-optical phase shifter and switch near 1550nm using tungsten disulfide $\left(W_{2}\right.$ ) deposited tapered fiber. Opt. Express 25, 17639-17649 (2017)

220. Chu, R. et al. Graphene decorated twin-core fiber Michelson interferometer for all-optical phase shifter and switch. Opt. Lett. 45, 177-180 (2020).

221. Hao, T., Chang, Z. S. \& Chiang, K. S. Externally pumped low-loss graphenebased fiber Mach-Zehnder all-optical switches with $\mathrm{mW}$ switching powers. Opt. Express 27, 4216-4225 (2019).

222. Wu, Q. et al. MZl-based all-optical modulator using MXene $\mathrm{Ti}_{3} \mathrm{C}_{2} \mathrm{~T}_{\mathbf{x}}(\mathrm{T}=\mathrm{F}, \mathrm{O}$, or $\mathrm{OH}$ ) deposited microfiber. Adv. Mater. Technol. 4, 1800532 (2019).

223. Zheng, J. L. et al. Few-layer phosphorene-decorated microfiber for all-optical thresholding and optical modulation. Adv. Optical Mater. 5, 1700026 (2017)

224. Guo, Q. B. et al. Boron nanosheets for efficient all-optical modulation and logic operation. Adv. Optical Mater. 7, 1900322 (2019).

225. Chai, Z. et al. Ultrafast all-optical switching. Adv. Optical Mater. 5, 1600665 (2017).

226. Romagnoli, M. et al. Graphene-based integrated photonics for nextgeneration datacom and telecom. Nat. Rev. Mater. 3, 392-414 (2018).

227. $\mathrm{Xu}, \mathrm{K}$. et al. High-speed traveling-wave modulator based on graphene and microfiber. J. Lightwave Technol. 36, 4730-4735 (2018).

228. Shah, M. K. et al. Graphene-assisted electroabsorption optical modulator using D-microfiber. IEEE J. Sel. Top. Quantum Electron. 23, 3400305 (2017).

229. Cheng, X. et al. Sandwiched graphene/hBN/graphene photonic crystal fibers with high electro-optical modulation depth and speed. Nanoscale $\mathbf{1 2}$ 14472-14478 (2020).

230. Dong, L. P. et al. All-fiber multifunctional electrooptic prototype device with a graphene/PMMA (poly(methyl methacrylate)) hybrid film integrated on coreless side-polished fibers. ACS Appl. Electron. Mater. 2, 447-455 (2020).

231. Chen, Y. et al. Demonstration of a microelectromechanical tunable FabryPérot cavity based on graphene-bonded fiber devices. Opt. Lett. 44, 1876-1879 (2019).

232. Buscema, M. et al. Photocurrent generation with two-dimensional van der Waals semiconductors. Chem. Soc. Rev. 44, 3691-3718 (2015).

233. Wang, F. et al. 2D library beyond graphene and transition metal dichalcogenides: a focus on photodetection. Chem. Soc. Rev. 47, 6296-6341 (2018).

234. Sun, X. W. et al. Broadband photodetection in a microfiber-graphene device Opt. Express 23, 25209-25216 (2015).

235. Chen, J. H. et al. Towards an all-in fiber photodetector by directly bonding few-layer molybdenum disulfide to a fiber facet. Nanoscale 9, 3424-3428 (2017).

236. Xiong, Y. F. et al. Broadband optical-fiber-compatible photodetector based on a graphene- $\mathrm{MoS}_{2}-\mathrm{WS}_{2}$ Heterostructure with a synergetic photogenerating mechanism. Adv. Electron. Mater. 5, 1800562 (2019).

237. Chen, J. H. et al. High-sensitivity optical-fiber-compatible photodetector with an integrated $\mathrm{CsPbBr}_{3}$-graphene hybrid structure. Optica 4, 835-838 (2017).

238. Xiong, Y. F. et al. Ultrahigh responsivity photodetectors of $2 \mathrm{D}$ covalent organic frameworks integrated on graphene. Adv. Mater. 32, 1907242 (2020).

239. Zhuo, L. Q. et al. A broadband all-fiber integrated graphene photodetector with CNT-enhanced responsivity. Nanoscale 12, 14188-14193 (2020).

240. Jin, Z. H. et al. Near-field coupled integrable two-dimensional InSe photosensor on optical fiber. ACS Nano 12, 12571-12577 (2018).

241. Koppens, F. H. L. et al. Photodetectors based on graphene, other twodimensional materials and hybrid systems. Nat. Nanotechnol. 9, 780-793 (2014). 
242. Allain, A. et al. Electrical contacts to two-dimensional semiconductors. Nat. Mater. 14, 1195-1205 (2015).

243. Guo, Q. S. et al. Efficient electrical detection of mid-infrared graphene plasmons at room temperature. Nat. Mater. 17, 986-992 (2018).

244. Buckley, D. J. et al. Frontiers of graphene and 2D material-based gas sensors for environmental monitoring. 2D Mater. 7, 032002 (2020).

245. Rodrigo, D. et al. Mid-infrared plasmonic biosensing with graphene. Science 349, 165-168 (2015)

246. Yu, R. W. et al. Nonlinear plasmonic sensing with nanographene. Phys. Rev. Lett. 117, 123904 (2016).

247. Xue, T. Y. et al. Ultrasensitive detection of miRNA with an antimonene-based surface plasmon resonance sensor. Nat. Commun. 10, 28 (2019).

248. Shivananju, B. N. et al. Graphene heterostructure integrated optical fiber Bragg grating for light motion tracking and ultrabroadband photodetection from $400 \mathrm{~nm}$ to $10.768 \mu \mathrm{m}$. Adv. Funct. Mater. 29, 1807274 (2019).

249. Sun, Q. Z. et al. Graphene-assisted microfiber for optical-power-based temperature sensor. IEEE Photonics Technol. Lett. 28, 383-386 (2015).

250. Hernaez, M. et al. Optical fibre sensors using graphene-based materials: a review. Sensors 17, 155 (2017)

251. Wu, Y. et al. Optical graphene gas sensors based on microfibers: a review. Sensors 18, 941 (2018).

252. Zhao, Y. et al. Review on the graphene based optical fiber chemical and biological sensors. Sens. Actuators B: Chem. 231, 324-340 (2016).

253. Liu, Y. X., Dong, X. C. \& Chen, P. Biological and chemical sensors based on graphene materials. Chem. Soc. Rev. 41, 2283-2307 (2012).

254. Wu, Y. et al. Hybrid graphene-microfiber waveguide for chemical gas sensing. IEEE J. Sel. Top. Quantum Electron. 20, 4400206 (2014).

255. Jiang, B. Q. et al. Graphene-coated tilted fiber-Bragg grating for enhanced sensing in low-refractive-index region. Opt. Lett. 40, 3994-3997 (2015).

256. Sridevi, $\mathrm{S}$. et al. Ultra sensitive $\mathrm{NO}_{2}$ gas detection using the reduced graphene oxide coated etched fiber Bragg gratings. Sens. Actuators B: Chem. 223, 481-486 (2016).

257. Yao, B. C. et al. All-optical Mach-Zehnder interferometric $\mathrm{NH}_{3}$ gas sensor based on graphene/microfiber hybrid waveguide. Sens. Actuators B: Chem. 194, 142-148 (2014)

258. Wu, Y. et al. Graphene-based D-shaped fiber multicore mode interferometer for chemical gas sensing. Opt. Lett. 39, 6030-6033 (2014).

259. Yu, C. B. et al. Graphene oxide deposited microfiber knot resonator for gas sensing. Optical Mater. Express 6, 727-733 (2016).

260. Hao, T. \& Chiang, K. S. Graphene-based ammonia-gas sensor using in-fiber mach-zehnder interferometer. IEEE Photonics Technol. Lett. 29, 2035-2038 (2017).

261. Yao, Q. F. et al. 2D plasmonic tungsten oxide enabled ultrasensitive fiber optics gas sensor. Adv. Optical Mater. 7, 1901383 (2019).

262. Wei, W. et al. Graphene-based long-period fiber grating surface plasmon resonance sensor for high-sensitivity gas sensing. Sensors 17, 2 (2016).

263. $\mathrm{Fu}, \mathrm{H}$. W. et al. Zinc oxide nanoparticle incorporated graphene oxide as sensing coating for interferometric optical microfiber for ammonia gas detection. Sens. Actuators B Chem. 254, 239-247 (2018).

264. Yao, B. C. et al. Partially reduced graphene oxide based FRET on fiber-optic interferometer for biochemical detection. Sci. Rep. 6, 23706 (2016).

265. Ma, J. et al. Fiber-optic ferrule-top nanomechanical resonator with multilayer graphene film. Opt. Lett. 39, 4769-4772 (2014).

266. Tan, Y. Z. et al. Optical fiber photoacoustic gas sensor with graphene nanomechanical resonator as the acoustic detector. IEEE J. Sel. Top. Quantum Electron. 23, 5600211 (2017)
267. Chen, C. Y. et al. Performance of monolayer graphene nanomechanical resonators with electrical readout. Nat. Nanotechnol. 4, 861-867 (2009).

268. Bunch, J. S. et al. Electromechanical resonators from graphene sheets. Science 315, 490-493 (2007)

269. Guo, B. 2D noncarbon materials-based nonlinear optical devices for ultrafast photonics. Chin. Opt. Lett. 16, 020004 (2018)

270. Liu, X. F., Guo, Q. B. \& Qiu, J. R. Emerging low-dimensional materials for nonlinear optics and ultrafast photonics. Adv. Mater. 29, 1605886 (2017).

271. Yamashita, S. Nonlinear optics in carbon nanotube, graphene, and related 2D materials. APL Photonics 4, 034301 (2019).

272. Bogusławski, J. et al. Graphene actively mode-locked lasers. Adv. Funct. Mater 28, 1801539 (2018)

273. Luo, Z. C. et al. $2 \mathrm{GHz}$ passively harmonic mode-locked fiber laser by a microfiber-based topological insulator saturable absorber. Opt. Lett. $\mathbf{3 8}$ 5212-5215 (2013).

274. Jiang, B. Q. et al. High-efficiency second-order nonlinear processes in an optical microfibre assisted by few-layer GaSe. Light.: Sci. Appl. 9, 63 (2020).

275. Wu, Y. et al. Four-wave mixing in a microfiber attached onto a graphene film. IEEE Photonics Technol. Lett. 26, 249-252 (2014).

276. Cox, J. D., Marini, A. \& de Abajo, F. J. G. Plasmon-assisted high-harmonic generation in graphene. Nat. Commun. 8, 14380 (2017)

277. Cox, J. D. \& de Abajo, F. J. G. Electrically tunable nonlinear plasmonics in graphene nanoislands. Nat. Commun. 5, 5725 (2014).

278. Liu, C. et al. Designed growth of large-size 2D single crystals. Adv. Mater. 32 2000046 (2020).

279. Coleman, J. N. Liquid Exfoliation of defect-free graphene. Acc. Chem. Res. 46, 14-22 (2013).

280. Amani, M. et al. Near-unity photoluminescence quantum yield in $\mathrm{MoS}_{2}$. Science 350, 1065-1068 (2015).

281. $\mathrm{Hu}, \mathrm{G}$. H. et al. Functional inks and printing of two-dimensional materials. Chem. Soc. Rev. 47, 3265-3300 (2018).

282. Jin, X. X. et al. 102 fs pulse generation from a long-term stable, inkjet-printed black phosphorus-mode-locked fiber laser. Opt. Express 26, 12506-12513 (2018).

283. Mirabelli, G. et al. Air sensitivity of $\mathrm{MoS}_{2}, \mathrm{MoSe}_{2}, \mathrm{MoTe}_{2}, \mathrm{HfS}_{2}$, and HfSe 2 . J. Appl. Phys. 120, 125102 (2016).

284. Edmonds, M. T. et al. Stability and surface reconstruction of topological insulator $\mathrm{Bi}_{2} \mathrm{Se}_{3}$ on exposure to atmosphere. J. Phys. Chem. C. $\mathbf{1 1 8}$ 20413-20419 (2014).

285. Zhang, K. L. et al. Two dimensional hexagonal boron nitride (2D-hBN): synthesis, properties and applications. J. Mater. Chem. C. 5, 11992-12022 (2017).

286. Gibertini, M. et al. Magnetic 2D materials and heterostructures. Nat. Nanotechnol. 14, 408-419 (2019).

287. Geim, A. K. \& Grigorieva, I. V. Van der Waals heterostructures. Nature 499 419-425 (2013).

288. Paik, E. Y. et al. Interlayer exciton laser of extended spatial coherence in atomically thin heterostructures. Nature 576, 80-84 (2019).

289. Sun, Z. Y. et al. Giant nonreciprocal second-harmonic generation from antiferromagnetic bilayer $\mathrm{Cr}_{3}$. Nature 572, 497-501 (2019).

290. Deng, Y. J. et al. Gate-tunable room-temperature ferromagnetism in twodimensional $\mathrm{Fe}_{3} \mathrm{GeTe}_{2}$. Nature 563, 94-99 (2018).

291. Dai, Z. G. et al. Artificial metaphotonics born naturally in two dimensions. Chem. Rev. 120, 6197-6246 (2020). 